\title{
PREDICTION OF MACHINING OPERATIONS AND SURFACE ROUGHNESS USING ARTIFICIAL NEURAL NETWORK
}

\author{
O. B. Abouelatta \\ Production Engineering and Mechanical Design Department, Faculty of Engineering, \\ Mansoura University, 35516 Mansoura, Egypt.
}

Received 11 February 2013, accepted 27 March 2013

\begin{abstract}
Surface roughness is considered as one of the most specified customer requirements in machining processes. For efficient use of machine tools, selection of machining process and determination of optimal cutting parameters (speed, feed and depth of cut) are required. Therefore, it is necessary to find a suitable way to select and to find optimal machining process and cutting parameters for a specified surface roughness values. In this work, machining process was carried out on AISI 1040 steel in dry cutting condition in a lathe, milling and grinding machines and surface roughness was measured. Forty five experiments have been conducted using varying speed, feed, and depth of cut in order to find the surface roughness parameters. This data has been divided into two sets on a random basis; 36 training data set and 9 testing data set. The training data set has been used to train different artificial neural network (ANN) models in order to predict machining processes and surface roughness parameter values through back propagation network. Experimental data collected from tests were used as input parameters of a neural network to identify the sensitivity among machining operations, cutting parameters and surface roughness. Selected indexes were used to design a suitable algorithm for the prediction of machining processes. A software was developed and implemented to predict the machining processes and surface roughness values. The results showed that the proposed models are capable of predicting machining operations, cutting parameters and surface roughness.
\end{abstract}

Keywords: Machining operations, Surface roughness, Neural network, Prediction.

\section{Introduction}

Increased demands for higher product quality, reliability, and manufacturing efficiency levels have imposed stringent requirements on automated product measurement and evaluation. The progress in the development of predictive models, based on cutting theory, has not yet met the objective of maintaining high quality production at low cost. The most essential cutting performance measures, such as, tool life, cutting force, roughness of the machined surface, energy consumption, ... etc., should be defined using experimental studies. Therefore, further improvement and optimization for the technological and economic performance of machining operations depend on a well-based experimental methodology. Hayajneh et al. [1], aimed to develop a better understanding of the effects of cutting parameters on the surface roughness and to build a multiple regression model. Suhail et al. [2], proposed a method for cutting parameters identification using multi adaptive network based fuzzy inference system (MANFIS). Their results showed that the MANFIS model can be used successfully for machinability data selection.

Journal of Engineering Sciences, Assiut University, Faculty of Engineering, Vol. 41, No. 3, May, 2013,E-mail address: jes@aun.edu.eg 
O. B. Abouelatta, Prediction of machining operations and surface roughness using artificial neural network, pp. 1021 - 1044

To deal with a nonlinear optimization problem which aims to minimize the unit production cost in multi-pass turnings, Xie and Guo [3] proposed a new approach, which combines genetic algorithms with a pass enumerating method. Computer simulation results showed that the optimization approach can find the better results than other algorithms proposed previously to significantly reduce the unit production cost. Grzesik and Wanat [4] provided a comprehensive analysis of part surface finish in continuous dry turning of hardened construction steel when using mixed alumina cutting tools. Their results showed that hard turning with mixed alumina tools produces specific surface profiles and microstructures, although the Ra roughness values of about $0.25 \mu \mathrm{m}$ can be comparable to those produced by finish grinding. Surface roughness has been modeled by Patrikar [5], based on power series and Fast Fourier Transform methods. A method based on neural networks has been used to model these surfaces to map the process parameters to roughness parameters.

In recent years, the trends were towards modeling of machining using artificial intelligence. Artificial neural networks are considered one of the important methods of artificial intelligence in the modeling of nonlinear problems like machining processes. ANN show good capability in prediction and optimization of machining processes compared with traditional methods. In turning operations, Luong and Spedding [6] described the application of neural-network technology to the selection of machining parameters and to the prediction of machining performance in metal cutting via an on-line implementation of the trained network using the $\mathrm{C}$ programming language.

A neural-network-based methodology was proposed by Kohli and Dixit [7], for predicting the surface roughness in a turning process by taking the acceleration of the radial vibration of the tool holder as feedback. They stated that their methodology was able to make accurate prediction of surface roughness by utilizing small sized training and testing datasets. ANN and multiple regression approaches were used by Asiltürk and Çunkaş [8], to model the surface roughness of AISI 1040 steel. The ANN model was used to estimate the surface roughness with high accuracy compared to the multiple regression model. Models for predicting surface roughness of AISI 1040 steel material using artificial neural networks and multiple regression were developed by Asiltürk [9]. Mean squared error of $0.00292 \%$ achieved using the developed ANN outperforms error rates reported in earlier studies and could also be considered admissible for real-time deployment of the developed ANN algorithm for robust prediction of the surface roughness in industrial settings.

In milling operations, multiple regression and ANN techniques were applied by Murthy and Rajendran [10], to predict the surface roughness. The results of the prediction models were quite close with experiment values. They reported that the feed rate was the most dominant factor in influencing surface roughness. The results also showed that the highest cutting speed, medium feed rate and medium depth of cut produces lowest surface roughness. An empirical approach using a statistical analysis was employed by Huang and Chen [11], to discover the proper cutting force in order to represent the uncontrollable

Journal of Engineering Sciences, Assiut University, Faculty of Engineering, Vol. 41, No. 3, May, 2013,E-mail address: jes@aun.edu.eg 
O. B. Abouelatta, Prediction of machining operations and surface roughness using artificial neural network, pp. 1021-1044

factors in end milling operations using an in-process neural network-based surface roughness prediction system.

Response surface model and an artificial neural network were developed by Erzurumlu and Oktem [12], to predict surface roughness values error on mold surfaces. The response surface model and an artificial neural network were compared with manufacturing problems such as computational cost, cutting forces, tool life, dimensional accuracy, etc. A new technique was developed by Wibowo and Desa [13], using hybridization of kernel principal component analysis based on nonlinear regression and GAs to estimate the optimum values of surface roughness. Their results showed that the presented technique gave more accurate prediction model than the ordinary linear regression's approach.

The need for precision components and parts in manufacturing industries has bought an increase in the need for finishing operations that can satisfy this demand. Grinding has the potential to meet these critical needs for accurate and economic means of finishing parts, and generate the required surface topography. An approach suggesting the combination of design of experiment method and ANN was developed by Fredj and Amamou [14]. The built ANNs showed low deviation from the training data, low deviation from the testing data and high sensibility to the inputs levels. The high prediction accuracy of the developed ANNs was confirmed by the good agreement with the results of empirical models developed by previous investigations. Mukherjee and Kumar Ray [15] attempted to provide a systematic methodology to develop a multivariate linear regression model, hypothesis testing for the influence of nonlinear terms to linear model, and accordingly selection of a suitable artificial neural network-based inferential model with improved prediction accuracy and control of grinding behavior.

The development of neural model-based control strategies for the optimization of an industrial aluminum substrate disk grinding process was described by Govindhasamy et al. [16], using a nonlinear autoregressive exogenous based neural network model. Preliminary plant investigations show that thickness defects can be reduced by $50 \%$ or more, compared to other schemes employed. Kumar and Choudhury [17] focused on the prediction of wheel wear and surface roughness using two techniques, namely design of experiments and neural network. Effect of process parameters, such as pulse current, duty ratio, wheel speed and grain size on output responses, namely, wheel wear and surface roughness of high speed steel were investigated experimentally.

This paper aims to reduce the huge number of two dimensional surface roughness parameters, to propose some indexes suitable for the prediction of machining processes and machining parameters based on surface roughness signature analysis using neural network.

\section{Experimental setup and procedure}

\subsection{Design of experiment}

Experiments have been performed in order to investigate the effects of machining parameters (speed, feed and depth of cut) on the surface finish of the machined surface.

Journal of Engineering Sciences, Assiut University, Faculty of Engineering, Vol. 41, No. 3, May, 2013,E-mail address: jes@aun.edu.eg 
1024

O. B. Abouelatta, Prediction of machining operations and surface roughness using artificial neural network, pp. 1021 - 1044

Forty five specimens made of AISI 1040 steel, $45 \mathrm{~mm}$ diameter with $10 \mathrm{~mm}$ in height, were used for experimentation using a turning (facing), milling and grinding machines. Chemical composition and mechanical properties of AISI 1040 steel are listed in Table 1 (a) and (b), respectively. The specimens were divided into three groups according to the machining operation; each has 15 specimens (12 for training and 3 for evaluation). Before employing machining process, each specimen was polished using sandpapers grade 1200 to eliminate the effect of previous cuts. All experiments have been done under dry machining environment. Several variables were put under close control including the machining condition (the same machine was used for all experimental work for each group), and the operator (the same operator machined all specimens for each machine).

Table 1. (a)

Chemical composition of AISI 1040 steel

\begin{tabular}{cccccc}
\hline \hline & \multicolumn{5}{c}{ Chemical composition (Wt. \%) } \\
Carbon & Iron & Manganese & Phosphorus & Sulphur & Others \\
\hline 0.37 & 98.8 & 0.7 & 0.035 & 0.045 & 0.05 \\
\hline \hline
\end{tabular}

Table 1. (b)

Mechanical properties of AISI 1040 steel

\begin{tabular}{cccc}
\hline \hline & \multicolumn{2}{c}{ Mechanical properties } & \\
Yield tensile strength & Ultimate tensile strength & Vickers Hardness & Modulus of \\
$\sigma_{\text {vield }}(\mathrm{MPa})$ & $\sigma_{\text {ultimate }}(\mathrm{MPa})$ & $(\mathrm{HV})$ & Elasticity $(\mathrm{GPa})$ \\
\hline 450 & 515 & 155 & 200 \\
\hline \hline
\end{tabular}

The first group was produced by lathe turning machine (Facing) under different cutting parameters. A cemented carbide cutting tool equipped with throwaway inserts was used in the experiments. The insert type was KPGN 160412 and the shank type was MSKNR/L $2525 \mathrm{M} 16$ for approach angle $\chi=35^{\circ}$. The second group was produced by a horizontal milling machine. A 3-inch $(\approx 80 \mathrm{~mm}$ outer diameter), 14-flute high-speed steel cutters and a $80 \mathrm{~mm}$ width was used in the experiments. The third group was machined by a surface grinder machine with electromagnetic chuck. The used cutting tool for grinding operations is aluminum oxide grinding wheels (38A60KK5VBE).

\subsection{Surface roughness measurements}

There is an industrial need for the measurement and classification of the topography of engineering surfaces. Two-dimensional (2D) analysis is fast, but limited in the usefulness of the results obtainable whilst the three-dimensional (3D) approach offers greater scope. Unfortunately, the data analysis step for 3D data characterization can be time-consuming, and often requires a skilled metrologist [18].

Journal of Engineering Sciences, Assiut University, Faculty of Engineering, Vol. 41, No. 3, May, 2013,E-mail address: jes@aun.edu.eg 
O. B. Abouelatta, Prediction of machining operations and surface roughness using artificial neural network, pp. 1021- 1044

In this investigation, a set of 2D surface roughness parameters, as well as profile and surface characteristics, such as the amplitude distribution functions, bearing area curves and symmetrical curves of geometrical contact obtained for the machined surface, were determined and analyzed. The surface roughness data were collected for each of the 45 samples for the machining conditions as defined in Tables 2-4.

A surface roughness measuring system consists of two major parts, hardware and software was used. The hardware consists of two main items as shown in Fig. 1. Mitutoyo SurfTest-SJ201 is a surface measuring instrument, used in contact method surface assessment.

Figures 2 and 3 show two special programs written in Matlab ${ }^{\mathrm{TM}}$, Prediction of Machining Process and Cutting Parameters (PMPCP) and Surface Roughness Calculation Program (SRCP) were used to give a full assessment of surface roughness parameters from the resulted surface profile, respectively. The $P M P C P$ program is used to measure, import and export surface profile and surface roughness data from Mitutoyo SurfTest-SJ201. By clicking the "Run $S R C P$ " button, the $S R C P$ program can be invoked to calculate more than 66 standard and non-standard surface roughness parameters as shown in Table 5 [19]. Three measurements for workpiece surface roughness were made and averaged for each test. Figure 4 shows surface texture and surface profile for some machining operations.

\section{Table 2.}

Cutting and measured roughness parameters turning operation

\begin{tabular}{|c|c|c|c|c|c|c|c|c|}
\hline \multirow[b]{2}{*}{ No. } & \multicolumn{3}{|c|}{ Cutting parameters ${ }^{*}$} & \multicolumn{3}{|c|}{ Surface roughness parameters ${ }^{* *}$} & \multicolumn{2}{|c|}{ Surface indexes } \\
\hline & $\begin{array}{c}f \\
\mathrm{~mm} / \mathrm{rev}\end{array}$ & $\begin{array}{c}s \\
r p m\end{array}$ & $\begin{array}{c}d_{c} \\
m m\end{array}$ & $\begin{array}{c}R_{a} \\
\mu m\end{array}$ & $\begin{array}{c}S \\
m m\end{array}$ & $\begin{array}{c}P_{c} \\
\text { Count }\end{array}$ & $\begin{array}{c}P_{c} / R_{a} \\
\text { Count } / \mu m\end{array}$ & $\begin{array}{c}S / R_{a} \\
\mathrm{~mm} / \mu \mathrm{m}\end{array}$ \\
\hline 1 & 0.16 & 140 & 0.3 & 7.05 & 112.2 & 72 & 10.2 & 15.9 \\
\hline 2 & 0.16 & 224 & 0.3 & 4.69 & 79.3 & 101 & 21.5 & 16.9 \\
\hline 3 & 0.16 & 560 & 0.3 & 6.77 & 115.4 & 70 & 10.3 & 17.1 \\
\hline 4 & 0.16 & 900 & 0.3 & 3.25 & 105.4 & 83 & 25.4 & 32.4 \\
\hline 5 & 0.16 & 560 & 0.1 & 8.01 & 116.0 & 71 & 8.9 & 14.5 \\
\hline 6 & 0.16 & 560 & 0.75 & 5.00 & 121.0 & 76 & 15.3 & 24.2 \\
\hline 7 & 0.16 & 560 & 1.5 & 2.94 & 75.9 & 107 & 36.3 & 25.8 \\
\hline 8 & 0.08 & 560 & 0.3 & 6.00 & 111.9 & 74 & 12.3 & 18.7 \\
\hline 9 & 0.23 & 560 & 0.3 & 4.41 & 89.0 & 94 & 21.3 & 20.2 \\
\hline 10 & 0.32 & 560 & 0.3 & 6.59 & 113.6 & 71 & 10.8 & 17.2 \\
\hline 11 & 0.46 & 560 & 0.3 & 6.38 & 95.1 & 82 & 12.9 & 14.9 \\
\hline 12 & 0.59 & 560 & 0.3 & 9.59 & 106.8 & 75 & 7.9 & 11.1 \\
\hline 13 & 0.26 & 710 & 1.25 & 5.22 & 120.0 & 68 & 13.0 & 23.0 \\
\hline 14 & 0.32 & 180 & 1.25 & 5.91 & 90.3 & 89 & 15.0 & 15.3 \\
\hline 15 & 0.52 & 450 & 1.75 & 6.03 & 95.2 & 84 & 13.9 & 15.8 \\
\hline
\end{tabular}

${ }^{*} f, s$, and $d_{c}$ are feed, rotational cutting speed and depth of cut, respectively.

${ }^{* *} R_{a}, S$, and $P_{c}$ are arithmetic roughness height, mean spacing of adjacent local peaks, and peak count, respectively. 
O. B. Abouelatta, Prediction of machining operations and surface roughness using artificial neural network, pp. 1021- 1044

Table 3.

Cutting and measured roughness parameters milling operation

\begin{tabular}{|c|c|c|c|c|c|c|c|c|}
\hline \multirow[b]{2}{*}{ No. } & \multicolumn{3}{|c|}{ Cutting parameters ${ }^{*}$} & \multicolumn{3}{|c|}{ Surface roughness parameters ${ }^{* *}$} & \multicolumn{2}{|c|}{ Surface indexes } \\
\hline & $\underset{\mathrm{mm} / \mathrm{min}}{f}$ & $\begin{array}{c}s \\
\text { rpm }\end{array}$ & $\begin{array}{c}d_{c} \\
m m\end{array}$ & $\begin{array}{c}R_{a} \\
\mu m\end{array}$ & $\begin{array}{c}S \\
m m\end{array}$ & $\begin{array}{c}P_{c} \\
\text { Count }\end{array}$ & $\begin{array}{c}P_{d} / R_{a} \\
\text { Count } / \mu m\end{array}$ & $\begin{array}{c}S / R_{a} \\
m m / \mu m\end{array}$ \\
\hline 1 & 28 & 157 & 0.2 & 3.41 & 120.7 & 67 & 19.8 & 35.4 \\
\hline 2 & 55 & 157 & 0.2 & 3.73 & 127.3 & 64 & 17.2 & 34.1 \\
\hline 3 & 157 & 157 & 0.2 & 4.15 & 135.6 & 63 & 15.1 & 32.7 \\
\hline 4 & 110 & 157 & 0.2 & 5.23 & 165.4 & 53 & 10.2 & 31.6 \\
\hline 5 & 140 & 157 & 0.2 & 3.58 & 126.0 & 67 & 18.6 & 35.2 \\
\hline 6 & 110 & 80 & 0.2 & 3.09 & 149.8 & 57 & 6.5 & 33.2 \\
\hline 7 & 41 & 157 & 0.45 & 3.66 & 179.3 & 47 & 18.4 & 48.6 \\
\hline 8 & 110 & 80 & 0.2 & 3.07 & 142.6 & 59 & 9.4 & 24.9 \\
\hline 9 & 110 & 80 & 0.1 & 2.99 & 121.2 & 67 & 12.8 & 49.0 \\
\hline 10 & 110 & 157 & 0.35 & 5.11 & 165.5 & 50 & 19.2 & 46.4 \\
\hline 11 & 110 & 157 & 0.25 & 5.06 & 147.1 & 62 & 22.3 & 40.6 \\
\hline 12 & 110 & 157 & 0.3 & 3.71 & 150.2 & 54 & 9.7 & 32.4 \\
\hline 13 & 280 & 439 & 0.4 & 6.19 & 205.6 & 40 & 12.2 & 29.1 \\
\hline 14 & 20 & 40 & 0.3 & 6.38 & 158.9 & 60 & 14.5 & 40.5 \\
\hline 15 & 110 & 157 & 0.15 & 3.93 & 123.5 & 65 & 16.5 & 31.5 \\
\hline
\end{tabular}

* $f, s$, and $d_{c}$ are feed, rotational cutting speed and depth of cut, respectively.

${ }^{* * *} R_{a}, S$, and $P_{c}$ are arithmetic roughness height, mean spacing of adjacent local peaks, and peak count, respectively.

\section{Table 4.}

Cutting and measured roughness parameters grinding operation

\begin{tabular}{|c|c|c|c|c|c|c|c|c|}
\hline \multirow[b]{2}{*}{ No. } & \multicolumn{3}{|c|}{ Cutting parameters } & \multicolumn{3}{|c|}{ Surface roughness parameters } & \multicolumn{2}{|c|}{ Surface indexes } \\
\hline & $\begin{array}{c}V_{w} \\
\mathrm{~m} / \mathrm{min}\end{array}$ & $\begin{array}{c}V_{c} \\
\mathrm{~m} / \mathrm{s}\end{array}$ & $\begin{array}{c}a_{e} \\
\mu m\end{array}$ & $\begin{array}{l}R_{a} \\
\mu m\end{array}$ & $\underset{\mathrm{mm}}{S}$ & $\begin{array}{c}P_{c} \\
\text { Count }\end{array}$ & $\begin{array}{c}P_{c} / R_{a} \\
\text { Count } / \mu m\end{array}$ & $\begin{array}{c}S / R_{a} \\
\mathrm{~mm} / \mu \mathrm{m}\end{array}$ \\
\hline 1 & 6 & 35 & 20 & 0.57 & 53.8 & 164 & 287.3 & 94.3 \\
\hline 2 & 6 & 35 & 30 & 0.83 & 49.3 & 166 & 200.2 & 59.5 \\
\hline 3 & 6 & 35 & 40 & 0.76 & 46.1 & 176 & 231.6 & 60.6 \\
\hline 4 & 6 & 35 & 50 & 0.84 & 51.5 & 157 & 185.9 & 61.0 \\
\hline 5 & 6 & 20 & 20 & 0.90 & 59.7 & 136 & 151.4 & 66.6 \\
\hline 6 & 6 & 20 & 30 & 1.00 & 53.1 & 155 & 155.2 & 53.0 \\
\hline 7 & 6 & 20 & 40 & 0.70 & 49.9 & 163 & 231.6 & 70.8 \\
\hline 8 & 6 & 20 & 50 & 0.69 & 50.5 & 168 & 241.1 & 72.8 \\
\hline 9 & 8.4 & 20 & 20 & 0.64 & 46.7 & 178 & 278.5 & 73.3 \\
\hline 10 & 7.2 & 35 & 20 & 0.66 & 47.8 & 169 & 257.4 & 72.7 \\
\hline 11 & 4.8 & 35 & 20 & 0.73 & 42.7 & 192 & 264.2 & 58.8 \\
\hline 12 & 3.6 & 20 & 20 & 0.65 & 48.3 & 167 & 256.5 & 74.3 \\
\hline 13 & 10 & 20 & 20 & 0.82 & 57.4 & 141 & 173.0 & 70.3 \\
\hline 14 & 40 & 35 & 60 & 0.78 & 46.3 & 176 & 225.4 & 59.4 \\
\hline 15 & 5 & 35 & 10 & 0.94 & 56.5 & 145 & 153.2 & 59.9 \\
\hline
\end{tabular}

${ }^{7} V_{w}, V_{c}$, and $a_{e}$ are grinding wheel surface speed, workpiece speed, depth of cut, respectively.

${ }^{* * *} R_{\omega}, S$, and $P_{c}$ are arithmetic roughness height, mean spacing of adjacent local peaks, and peak count, respectively.

Journal of Engineering Sciences, Assiut University, Faculty of Engineering, Vol. 41, No. 3, May, 2013,E-mail address: jes@aun.edu.eg 


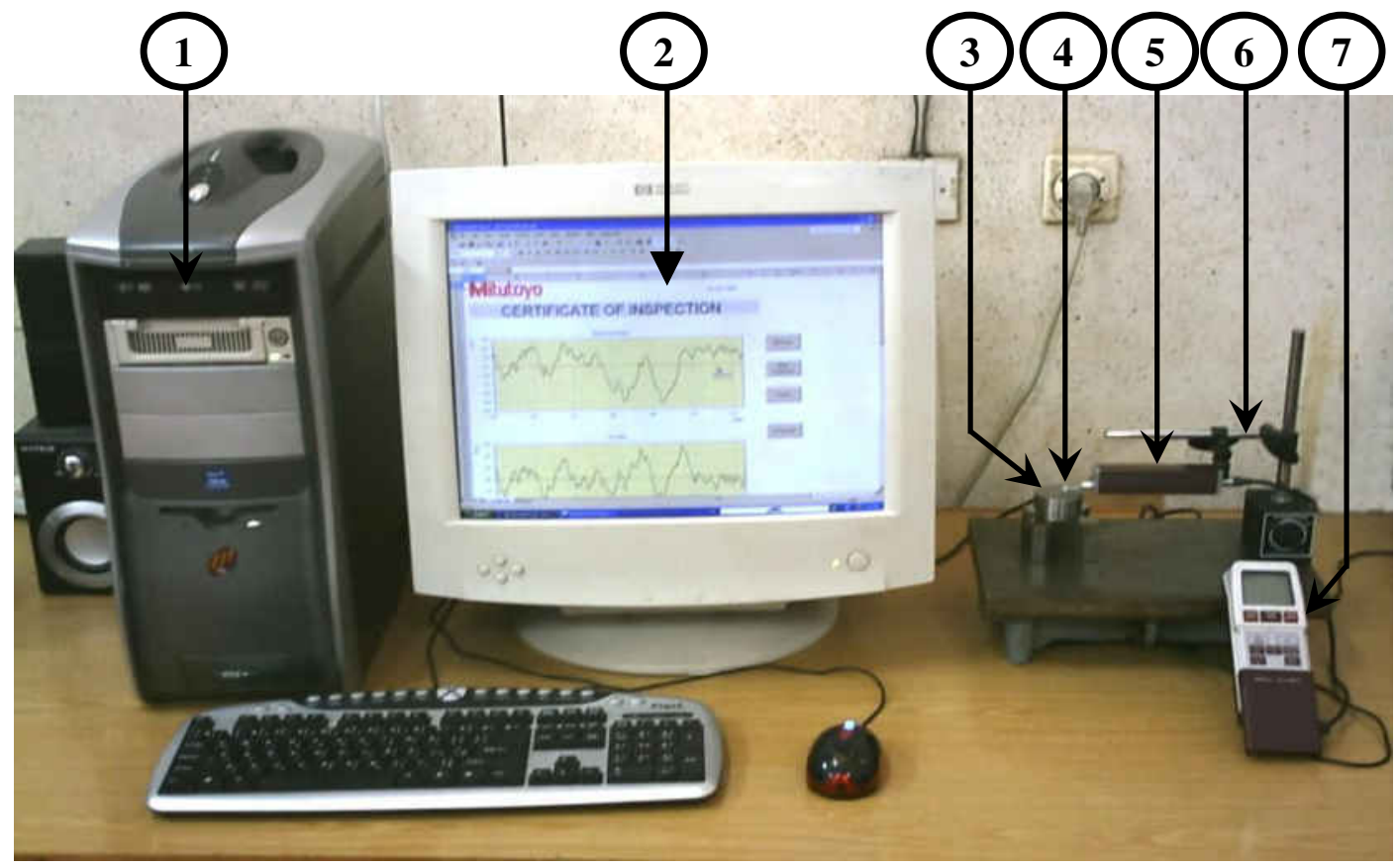
1. Personal computer
2. Mitutoyo interface program
3. Specimen
4. Stylus
5. SurfTest-SJ201 driving unit
6. Holder
7. SurfTest-SJ201 control unit

Fig. 1. SurfTest-SJ201 and personal computer (PC)

\section{Analysis procedure}

\subsection{Analysis of surface roughness parameters}

In recent years there has been a proliferation of parameters with which to specify surface texture. Some of these parameters are useful, but most are not [20]. The result of this rash is confusion and expense. So, a measure of the strength of the linear relationship between roughness parameters were applied using MS Office Excel to reduce the number of surface roughness parameters used in surface analysis. Table 6 shows a part of correlation coefficient between surface roughness parameter. Then, the surface roughness parameters regrouped with that having a strong correlation coefficient $(R \geq 0.90)$. The results of this analysis are grouped in six categories (I-VI) and listed in Table 7. The first group, for example, includes $R_{\omega}, R_{q}, R_{p}, R_{v}$, $R_{b} N_{\text {Peaks }}, A C F_{10 \%} A C F, W F$ roughness parameters. This means that any roughness parameter within group (I) is enough to study the effect of cutting parameter, for example, on the resulting surface roughness. The same could be applied for other groups (II-VI), i.e. any roughness parameter within the same group could be used to represent the effect of cutting parameter.

Journal of Engineering Sciences, Assiut University, Faculty of Engineering, Vol. 41, No. 3, May, 2013,E-mail address: jes@aun.edu.eg 
1028

O. B. Abouelatta, Prediction of machining operations and surface roughness using artificial neural network, pp. 1021 - 1044

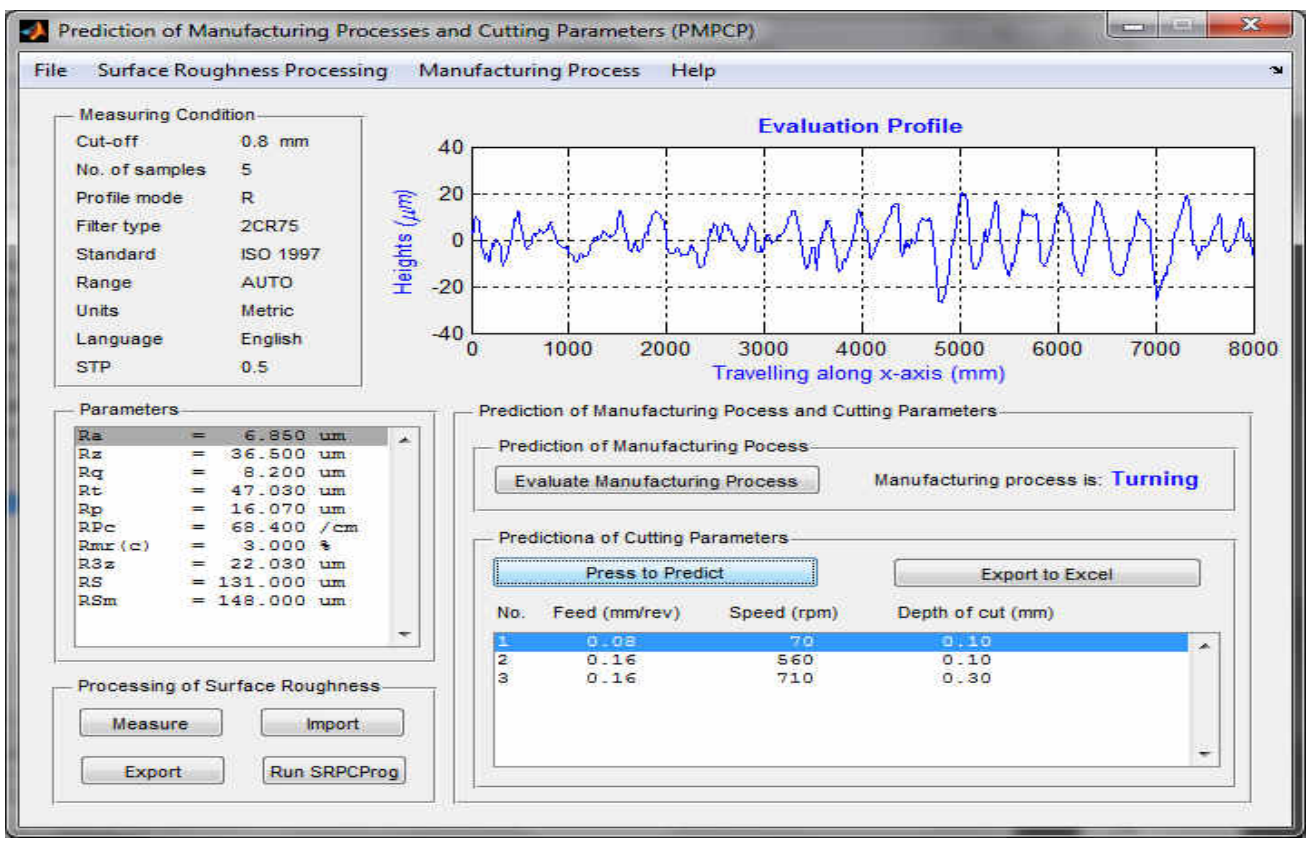

Fig. 2. $P M P C P$, Prediction of Machining Operations and Cutting Parameters Program

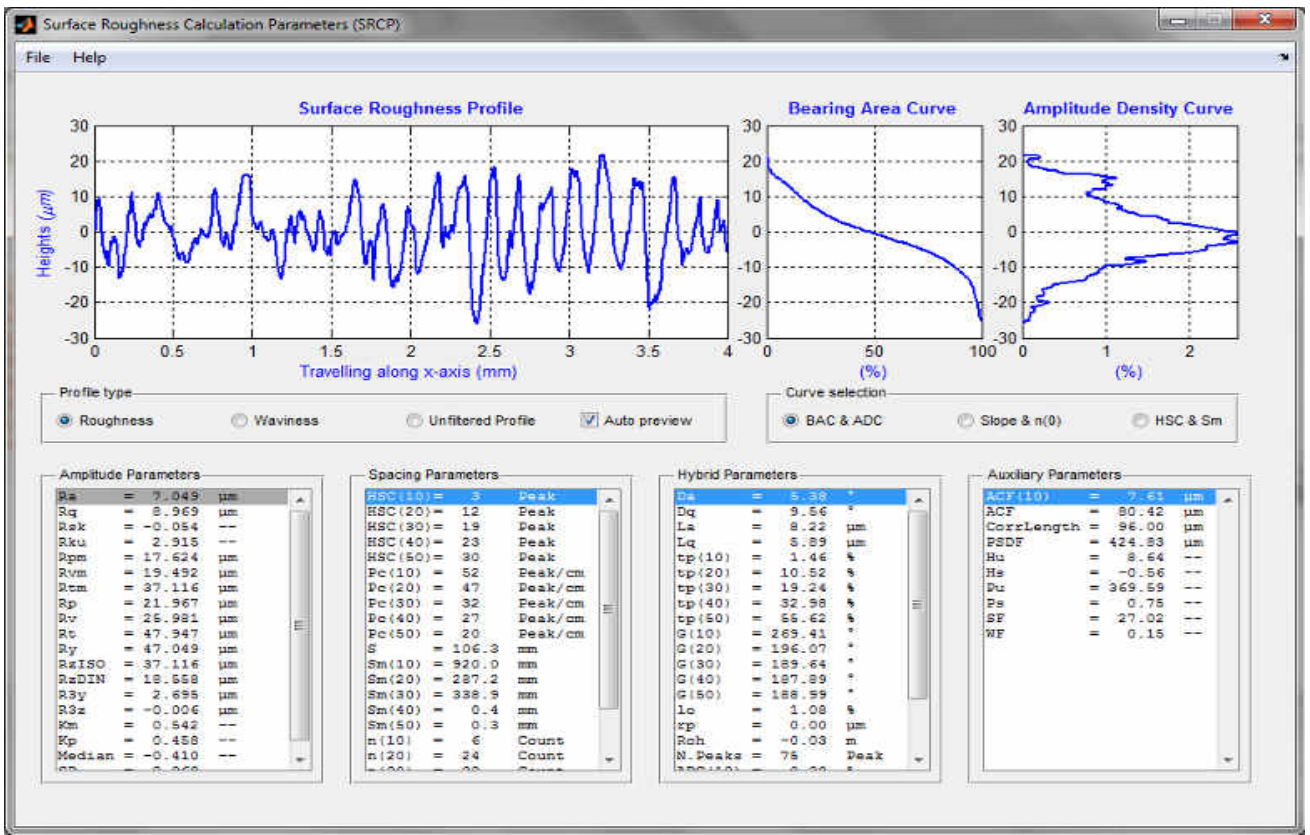

Fig. 3. SRCP, Surface Roughness Calculation Program

Journal of Engineering Sciences, Assiut University, Faculty of Engineering, Vol. 41, No. 3, May, 2013,E-mail address: jes@aun.edu.eg 
O. B. Abouelatta, Prediction of machining operations and surface roughness using artificial neural network, pp. 1021- 1044

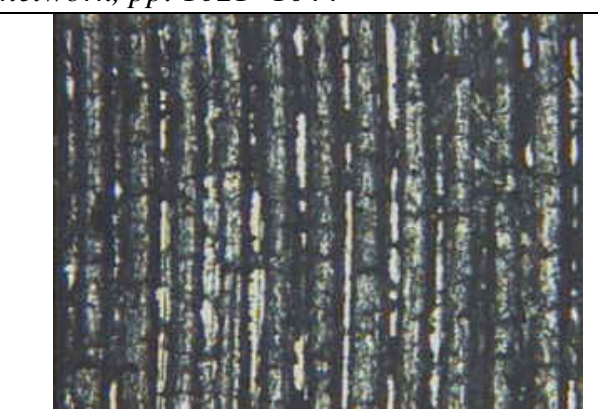

(a)

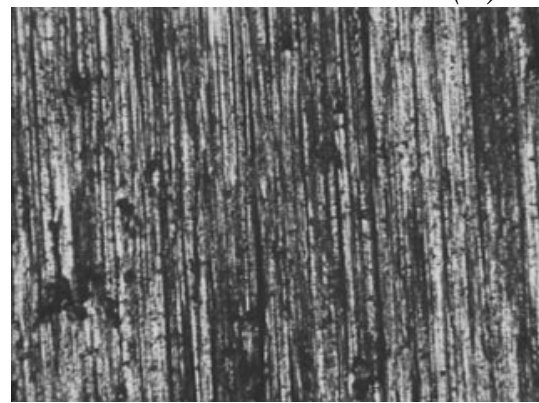

(b)

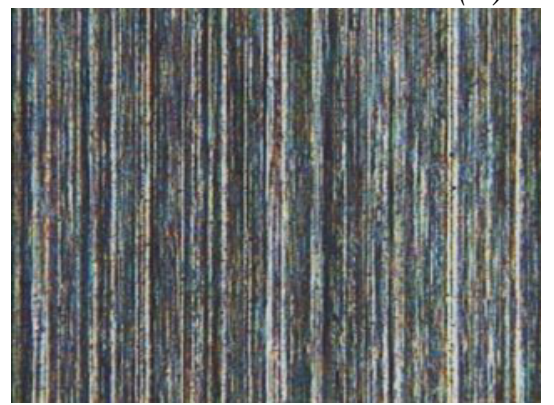

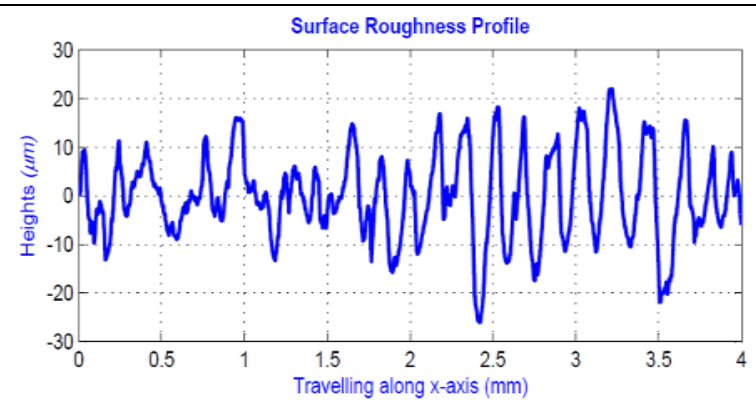

Turned workpiece No. (1)

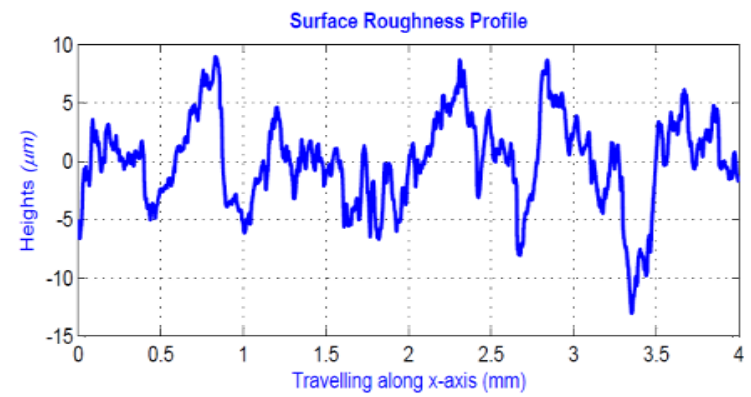

Milled workpiece No. (5)

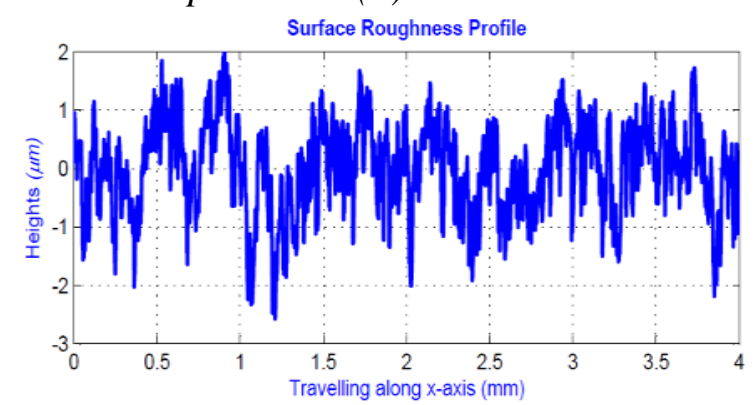

(c) Ground workpiece No. (6)

Fig. 4. Samples of surface texture and surface profile for some machining processes

\subsection{Analysis of machining operation signature}

The values of amplitude, spacing and hybrid parameters of surfaces manufactured by different machining operations are overlapped significantly. Even if one machining operation results in a similar surface pattern, it produces surfaces whose amplitude, spacing and hybrid parameters vary widely depending on the machining conditions. Thus, it is difficult to identify surface signature of different machining operations by investigating the magnitudes of the parameters [21]. It is considered here that a good method of classifying machining processes is to use indexes rather than absolute physical quantities. This section provides evidence of the relationships between machining

Journal of Engineering Sciences, Assiut University, Faculty of Engineering, Vol. 41, No. 3, May, 2013,E-mail address: jes@aun.edu.eg 
1030

O. B. Abouelatta, Prediction of machining operations and surface roughness using artificial neural network, pp. 1021 - 1044

operations and some derived indexes. However by examining these indexes, it is possible to identify different classes of machining operations.

Table 5. (a)

Standard and non-standard surface roughness parameters

\begin{tabular}{|c|c|c|c|c|}
\hline Surface roughness parameter & Turning & Milling & Grinding & Units \\
\hline$\overline{\text { Arithmetic average roughness, } R_{a}}$ & 5.86 & 4.22 & 0.78 & $\mu \mathrm{m}$ \\
\hline Geometric average roughness, $R_{q}$ & 7.15 & 5.34 & 0.98 & $\mu \mathrm{m}$ \\
\hline Skewness, $R_{s k}$ & 0.10 & -0.53 & -0.27 & ----- \\
\hline Kurtosis, $R_{k u}$ & 2.82 & 3.56 & 3.15 & ----- \\
\hline Mean of $R_{p i}$ values, $R_{p m}$ & 14.77 & 8.56 & 1.95 & $\mu \mathrm{m}$ \\
\hline Mean of $R_{v i}$ values, $R_{v m}$ & 12.32 & 10.53 & 2.58 & $\mu \mathrm{m}$ \\
\hline Mean of $R_{t i}$ values, $R_{t m}$ & 27.09 & 19.09 & 4.53 & $\mu \mathrm{m}$ \\
\hline Maximum height, $R_{p}$ & 18.41 & 11.91 & 2.52 & $\mu \mathrm{m}$ \\
\hline Maximum depth, $R_{v}$ & 17.19 & 16.27 & 3.45 & $\mu \mathrm{m}$ \\
\hline Maximum peak to valley height, $R_{t}$ & 35.60 & 28.18 & 5.98 & $\mu \mathrm{m}$ \\
\hline Largest $R_{t i}$ value, $R_{y}$ & 33.90 & 26.34 & 5.51 & $\mu \mathrm{m}$ \\
\hline Ten-point height, $R_{z I S O}$ & 27.09 & 19.09 & 4.53 & $\mu \mathrm{m}$ \\
\hline Ten-point height, $R_{z D I N}$ & 13.54 & 9.54 & 2.27 & $\mu \mathrm{m}$ \\
\hline Mean of $R_{3 y}, R_{3 z}$ & 7.42 & 3.14 & 0.32 & $\mu \mathrm{m}$ \\
\hline Fullness grade, $K_{m}$ & 0.48 & 0.58 & 0.58 & ----- \\
\hline Emptiness grade, $K_{p}$ & 0.52 & 0.42 & 0.42 & ----- \\
\hline Median & -0.57 & 0.52 & 0.05 & $\mu \mathrm{m}$ \\
\hline Standard Deviation, SD & 7.15 & 5.34 & 0.98 & $\mu m$ \\
\hline High spot count at $10 \%, H S C_{10 \%}$ & 3.87 & 5.33 & 10.94 & Peak \\
\hline High spot count at $30 \%, H_{S C} C_{30 \%}$ & 15.29 & 19.40 & 72.91 & Peak \\
\hline High spot count at $50 \%, H_{5 C}$ & 20.11 & 17.04 & 84.42 & Peak \\
\hline Peak count at $10 \%, P_{\text {clo\% }}$ & 33.44 & 28.94 & 125.26 & Peak/cm \\
\hline Peak count at $30 \%, P_{c 30 \%}$ & 21.55 & 15.61 & 42.51 & Peak/cm \\
\hline Peak count at $50 \%, P_{c 50 \%}$ & 12.72 & 8.17 & 17.31 & Peak/cm \\
\hline Mean spacing of adjacent local peaks, $S$ & 103.1 & 147.9 & 51.1 & $\mathrm{~mm}$ \\
\hline Mean spacing between profile peaks, $S_{m 10 \%}$ & 433.9 & 601.7 & 589.0 & $\mathrm{~mm}$ \\
\hline Mean spacing between profile peaks, $S_{m 30 \%}$ & 156.4 & 132.1 & 168.9 & $\mathrm{~mm}$ \\
\hline Mean spacing between profile peaks, $S_{m 50 \%}$ & 1.2 & 0.6 & 144.9 & $\mathrm{~mm}$ \\
\hline No. of intersections of the profile at $10 \%, n_{(0) 10 \%}$ & 7.8 & 10.9 & 21.9 & Count \\
\hline No. of intersections of the profile at $30 \%, n_{(0) 10 \%}$ & 31.0 & 39.5 & 146.3 & Count \\
\hline No. of intersections of the profile at $50 \%, n_{(0) 50 \%}$ & 41.3 & 35.4 & 170.0 & Count \\
\hline Number of inflection points, $g$ & 4706.7 & 4667.3 & 3506.4 & Count \\
\hline Average slope, $\Delta_{a}$ & 9.33 & 8.43 & 8.40 & degree \\
\hline Root mean slope, $\Delta_{q}$ & 13.78 & 13.08 & 14.62 & degree \\
\hline Average wavelength, $\lambda_{a}$ & 5.67 & 4.50 & 0.77 & $\mu \mathrm{m}$ \\
\hline Root mean wavelength, $\lambda_{a}$ & 4.60 & 3.24 & 0.48 & $\mu \mathrm{m}$ \\
\hline Bearing ratio at $10 \%, t_{p 10 \%}$ & 1.94 & 3.41 & 1.77 & $\%$ \\
\hline Bearing ratio at $30 \%, t_{p 30 \%}$ & 16.14 & 28.19 & 22.93 & $\%$ \\
\hline Bearing ratio at $50 \%, t_{p 50 \%}$ & 45.92 & 66.02 & 66.25 & $\%$ \\
\hline
\end{tabular}

Journal of Engineering Sciences, Assiut University, Faculty of Engineering, Vol. 41, No. 3, May, 2013,E-mail address: jes@aun.edu.eg 
O. B. Abouelatta, Prediction of machining operations and surface roughness using artificial neural network, pp. $1021-1044$

\section{Table 5. (b)}

Standard and non-standard surface roughness parameters (Continued)

\begin{tabular}{lcccc}
\hline \hline \multicolumn{1}{c}{ Surface roughness parameter } & Turning & Milling & Grinding & Units \\
\hline Profile slope at line 10\%, $\gamma_{10 \%}$ & 189.0 & 185.6 & 204.1 & degree \\
Profile slope at line $30 \%, \gamma_{30 \%}$ & 193.6 & 195.5 & 182.8 & degree \\
Profile slope at line $50 \%, \gamma_{50 \%}$ & 193.2 & 193.3 & 181.5 & degree \\
Relative length of the profile, $l_{o}$ & 1.04 & 1.02 & 1.01 & $\mu \mathrm{m}$ \\
Peak count, $r_{p}$ & 81 & 58 & 162 & Count \\
Amplitude Density Curve at 10\%, ADC $10 \%$ & 0.28 & 0.55 & 0.33 & $\%$ \\
Amplitude Density Curve at 30\%, ADC $C_{30 \%}$ & 1.10 & 1.80 & 1.81 & $\%$ \\
Amplitude Density Curve at $50 \%, A D C_{50 \%}$ & 1.61 & 1.56 & 1.99 & $\%$ \\
Autocorrelation function, ACF & 56.17 & 31.22 & 0.99 & ----- \\
Correlation Length, CL & 211.6 & 355.0 & 334.0 & $\mu \mathrm{m}$ \\
Power spectrum density function, $P S D F$ & 183.0 & 82.2 & 65.6 & ----- \\
Roughness height uniformity, $H_{u}$ & 6.86 & 4.77 & 0.92 & ----- \\
Roughness height skewness, $H_{s}$ & -0.54 & 0.49 & 0.03 & ----- \\
Roughness pitch uniformity, $P_{u}$ & 451.3 & 450.3 & 502.2 & ---- \\
Roughness pitch skewness, $P_{s}$ & 0.79 & 0.95 & 21.52 & ----- \\
Steepness factor of the profile, $S F$ & 13.62 & 9.45 & 0.58 & ----- \\
Waviness factor of the profile, $W F$ & 0.20 & 0.27 & 1.37 & ----- \\
\hline \hline
\end{tabular}

First of all, a paired of surface roughness parameters were selected to study its relation with the machining operations. The selected paired of surface roughness parameters, which called index, is based on some trails except for the condition of their poor correlation coefficient with each other. Then, by plotting this pair of surface roughness parameters, the machining operations can be partially or completely separated. If an approximate zone of a machining operations can be got based on the proposed indexes, the efficiency of the identification can be assessed. It was found that $P_{c} / R_{a}$ and $S / R_{a}$ indexes satisfy the condition of determining an approximate zone.

The machining operation zones which enclose the values of the indexes obtained under different cutting parameters are evident from Figs. 5 and 6. It was found that turning (Facing), milling and ground surfaces are distinctively separated from other machining operations. In general, turned surfaces have the largest $P_{d} / R_{a}$ index and $S / R_{a}$ index, whereas grinding surfaces have the lowest $P_{d} / R_{a}$ index and $S / R_{a}$ index. Milling surfaces have the second largest $P_{d} / R_{a}$ index and $S / R_{a}$ index.

Journal of Engineering Sciences, Assiut University, Faculty of Engineering, Vol. 41, No. 3, May, 2013,E-mail address: jes@aun.edu.eg 
O. B. Abouelatta, Prediction of machining operations and surface roughness using artificial neural network, pp. 1021- 1044

\section{Table 6.}

Correlation coefficient between surface roughness parameters (Correlation coefficients greater than 0.9 are highlighted with bold).

\begin{tabular}{lccccccccccc}
\hline \hline Parameters & $R_{p m}$ & $R_{t m}$ & $R_{p}$ & $R_{t}$ & $R_{y}$ & $R_{z I S O}$ & $R_{a}$ & $K_{m}$ & $K_{p}$ & Med & $S D$ \\
\hline$R_{z D I N}$ & $\mathbf{0 . 9 4 2}$ & $\mathbf{1 . 0 0 0}$ & 0.780 & 0.715 & $\mathbf{0 . 9 1 5}$ & $\mathbf{1 . 0 0 0}$ & 0.750 & 0.436 & 0.436 & 0.451 & 0.750 \\
$R_{q}$ & 0.652 & 0.750 & $\mathbf{0 . 9 4 8}$ & $\mathbf{0 . 9 5 2}$ & 0.772 & 0.769 & $\mathbf{0 . 9 9 8}$ & 0.314 & 0.314 & 0.633 & $\mathbf{1 . 0 0 0}$ \\
$R_{s k}$ & 0.595 & 0.421 & 0.556 & 0.269 & 0.265 & 0.424 & 0.435 & $\mathbf{0 . 9 3 5}$ & $\mathbf{0 . 9 3 5}$ & 0.770 & 0.398 \\
$R_{p m}$ & & $\mathbf{0 . 9 4 2}$ & 0.713 & 0.550 & 0.762 & $\mathbf{0 . 9 3 9}$ & 0.665 & 0.657 & 0.657 & 0.621 & 0.652 \\
$R_{v m}$ & & 0.874 & 0.712 & 0.803 & $\mathbf{0 . 9 4 3}$ & 0.878 & 0.714 & 0.025 & 0.025 & 0.110 & 0.735 \\
$R_{t m}$ & & & 0.780 & 0.715 & $\mathbf{0 . 9 1 5}$ & $\mathbf{1 . 0 0 0}$ & 0.750 & 0.436 & 0.436 & 0.451 & 0.750 \\
$R_{p}$ & & & $\mathbf{0 . 9 3 2}$ & 0.820 & 0.795 & $\mathbf{0 . 9 4 6}$ & 0.488 & 0.488 & 0.647 & $\mathbf{0 . 9 4 8}$ \\
$R_{v}$ & & & & $\mathbf{0 . 9 1 9}$ & 0.737 & 0.550 & 0.775 & 0.243 & 0.243 & 0.159 & 0.808 \\
$R_{t}$ & & & & & 0.843 & 0.732 & $\mathbf{0 . 9 3 3}$ & 0.150 & 0.150 & 0.446 & $\mathbf{0 . 9 5 2}$ \\
$R_{y}$ & & & & & & $\mathbf{0 . 9 1 8}$ & 0.751 & 0.228 & 0.228 & 0.276 & 0.772 \\
$R_{a}$ & & & & & & & 0.354 & 0.354 & 0.664 & $\mathbf{0 . 9 9 8}$ \\
$K_{m}$ & & & & & & & & & & 0.728 & 0.314 \\
\hline \hline
\end{tabular}

Table 7.

Grouping of a highly correlated surface roughness parameters.

\begin{tabular}{|c|c|c|c|c|c|c|c|c|}
\hline \multirow{2}{*}{$\begin{array}{l}\text { Group } \\
\text { No. } \\
\end{array}$} & \multicolumn{2}{|l|}{ Turning (Facing) } & \multicolumn{2}{|l|}{ Milling } & \multicolumn{2}{|l|}{ Grinding } & \multicolumn{2}{|l|}{ All Processes } \\
\hline & Parameters & No. & Parameters & No. & Parameters & No. & Parameters & No. \\
\hline I & $\begin{array}{l}R_{o}, R_{q}, R_{p}, R_{v}, R_{t}, \\
N_{\text {Peaks }}, A C F_{10 \%}, \\
\quad A C F, W F\end{array}$ & 9 & $\begin{array}{c}R_{a}, R_{q}, R_{p m}, R_{t m}, \\
R_{t}, R_{z I S O}, R_{z D I N}, \\
A C F_{10 \%}, A C F, H_{u}\end{array}$ & 10 & $\begin{array}{c}R_{a}, R_{q}, R_{p m}, R_{t m}, R_{v}, \\
R_{t}, R_{y}, R_{z I S O}, R_{z D I N}, \\
A C F_{10 \%}, A C F, H_{u}\end{array}$ & 12 & $\begin{array}{l}R_{a}, R_{q}, R_{p}, R_{v}, R_{t}, \\
N_{\text {Peaks }}, A C F_{10 \%}, \\
\text { ACF }, W F\end{array}$ & 9 \\
\hline II & $\begin{array}{l}R_{s k}, K_{m}, K_{p}, t p_{40 \%} \\
t p_{50 \%}, A D C_{40 \%}\end{array}$ & 6 & $R_{s k}, t p_{40 \%}, t p_{50 \%}$ & 3 & $A D C_{30 \%}, t p_{30 \%}$ & 4 & $t p_{40 \%}, t p_{50 \%}$ & 2 \\
\hline III & $\begin{array}{l}R_{z D I N}, R_{p m}, R_{t m}, R_{y}, \\
R_{z I S O}, H_{w}, R_{v m}\end{array}$ & 7 & $\begin{array}{l}R_{z D I N}, R_{p m w}, R_{t m}, R_{y}, \\
\quad R_{z I S O}, H_{w}, R_{v m}\end{array}$ & 7 & $\begin{array}{c}R_{z D I N}, R_{p m}, R_{t m}, R_{y} \\
R_{z I S O}, H_{w}, R_{v m}\end{array}$ & 7 & $\begin{array}{l}R_{z D I N}, R_{p m}, R_{t m}, R_{y}, \\
\quad R_{z I S O}, H_{w} R_{v m}\end{array}$ & 7 \\
\hline IV & $\begin{array}{c}P_{c 10 \%}, P_{c 20 \%}, P_{c 30 \%}, \\
P_{c 40 \%}, P_{c 50 \%} \\
H S C_{10 \%}, N_{\text {Intrlo\% }}, \\
H S C_{20 \%}, N_{\text {Intr } 20 \%}\end{array}$ & 5 & $\begin{array}{c}P_{c 10 \%,}, P_{c 20 \%,} \\
P_{c 30 \%}, P_{c 40 \%}, P_{c 50 \%} \\
H S C_{10 \%}, N_{\text {Intr } 10 \%}, \\
H S C_{20 \%}, N_{\text {Intr } 20 \%},\end{array}$ & 5 & $\begin{array}{c}P_{c 10 \%}, P_{c 20 \%}, P_{c 30 \%}, \\
P_{c 40 \%}, P_{c 50 \%} \\
H S C_{10 \%}, N_{\text {Intr } 10 \%}, \\
H S C_{20 \%}, N_{\text {Intr } 20 \%}\end{array}$ & 5 & $\begin{array}{c}P_{c 10 \%,}, P_{c 20 \%}, \\
P_{c 30 \%}, P_{c 40 \%}, P_{c 50 \%} \\
H S C_{10 \%}, N_{I n t r I 0 \%}, \\
H S C_{20 \%}, N_{l t t r 20 \%}\end{array}$ & 5 \\
\hline V & $\begin{array}{l}H S C_{30 \%}, N_{\text {Intr } 30 \%} \\
H S C_{40 \%}, N_{\text {Intr } 40 \%} \\
H S C_{50 \%}, N_{\text {Intr } 50 \%}\end{array}$ & 10 & $\begin{array}{l}H S C_{30 \%}, N_{\text {Intr } 30 \%}, \\
H S C_{40 \%}, N_{\text {Intr } 40 \%}, \\
H S C_{50 \%}, N_{\text {Int } 50 \%}\end{array}$ & 10 & $\begin{array}{l}H S C_{30 \%}, N_{\text {Int } 30 \%} \\
H S C_{40 \%}, N_{\text {Int } 40 \%}, \\
H S C_{50 \%}, N_{\text {Intr } 50 \%}\end{array}$ & 10 & $\begin{array}{l}H S C_{30 \%}, N_{\text {Intr } 30 \%}, \\
H S C_{40 \%}, N_{\text {Intr } 40 \%}, \\
H S C_{50 \%}, N_{\text {Intr } 50 \%}\end{array}$ & 10 \\
\hline VI & $\Delta_{a}, \Delta_{q}, \lambda_{a}, \lambda_{q}$ & 4 & $\Delta_{a}, \Delta_{q}, \lambda_{a}, \lambda_{q}$ & 4 & $\Delta_{a}, \Delta_{q}, \lambda_{a}, \lambda_{q}$ & 4 & $\Delta_{a}, \Delta_{q}, \lambda_{a}, \lambda_{q}$ & 4 \\
\hline
\end{tabular}

Correlation coefficients are equal or more than $0.90(P=0.05)$.

Each machining operation can be deduced in an area of ellipse as shown in Figs. 5 and 6. An ellipse is a smooth closed curve which is symmetric about its horizontal and vertical axes. The distance between antipodal points on the ellipse, or pairs of points whose midpoint is at the center of the ellipse, is maximum along the major axis or transverse

Journal of Engineering Sciences, Assiut University, Faculty of Engineering, Vol. 41, No. 3, May, 2013,E-mail address: jes@aun.edu.eg 
O. B. Abouelatta, Prediction of machining operations and surface roughness using artificial neural network, pp. 1021 - 1044

diameter, and a minimum along the perpendicular minor axis or conjugate diameter [22]. An ellipse in general position can be expressed parametrically as the path of a point $(X(t)$, $Y(t))$, where:

$$
\begin{aligned}
& X(t)=X c+a \cos (t) \cos (\varphi)-b \sin (t) \sin (\varphi) \\
& Y(t)=Y c+a \cos (t) \cos (\varphi)+b \sin (t) \sin (\varphi)
\end{aligned}
$$

as the parameter $t$ varies from 0 to $2 \pi$. Here $\left(X_{c}, Y_{c}\right)$ is the center of the ellipse, and $\varphi$ is the angle between the $X$-axis and the major axis of the ellipse. Table 8 lists the ellipse parameters $\left(X_{c}, Y_{c}, a, b\right.$ and $\left.\varphi\right)$ for different machining processes.

The range of the $P_{d} / R_{a}$ and $S / R_{a}$ indexes for different machining processes are given in Figs. 7-9, respectively. Although the conventional surface roughness parameters listed in Tables 2-4 vary considerably for each kind of surface, it is not difficult to observe from the figures that the indexes identify the different machining operations clearly. In addition, some general conclusions for the relationship between the indexes and machining operations can be drawn here with the help of Figs. 7-9. The indexes may also vary in range, see Table 2-4. It can be observed that if surfaces have clearly different surface roughness or height distributions, the distribution zones of the indexes of the surfaces would be distinctively separated. Moreover, the variation of the indexes of one kind of surface indicates differences of surface features although the surfaces may have similar surface roughness or height distributions.

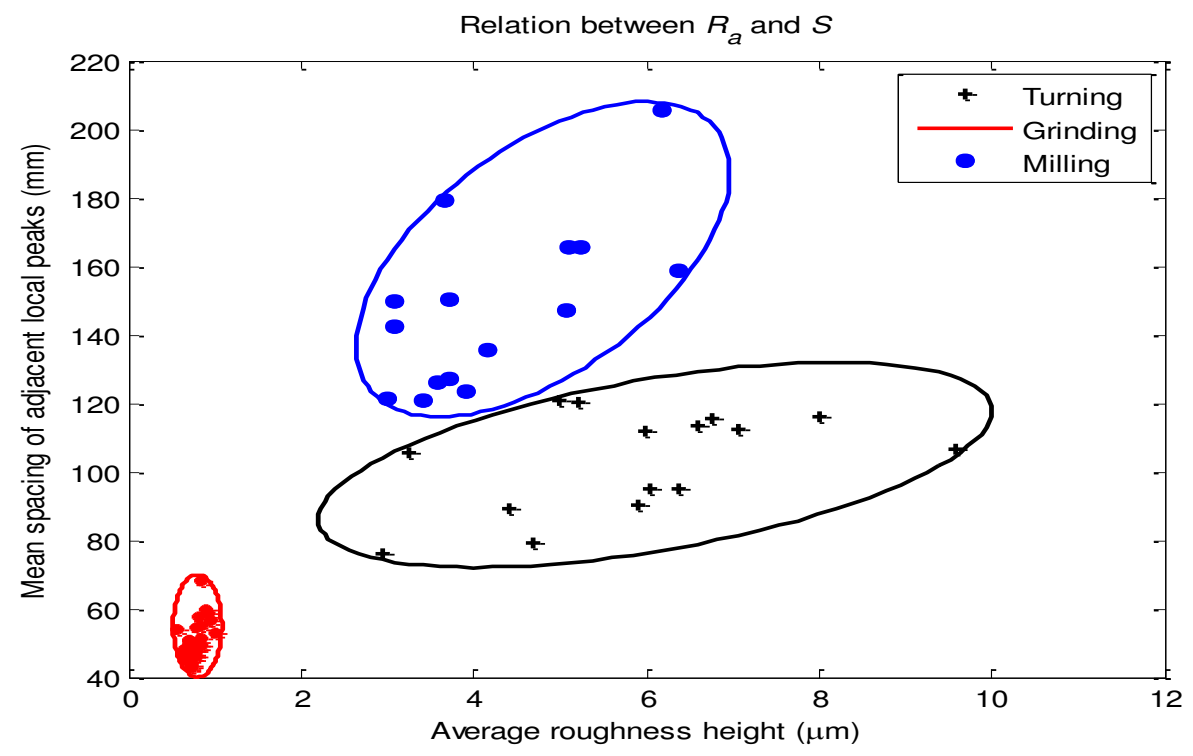

Fig. 5. A diagram of average roughness height $\left(R_{a}\right)$ versus mean spacing of adjacent local peaks $(S)$ of typical machining operations

Journal of Engineering Sciences, Assiut University, Faculty of Engineering, Vol. 41, No. 3, May, 2013,E-mail address: jes@aun.edu.eg 
O. B. Abouelatta, Prediction of machining operations and surface roughness using artificial neural network, pp. 1021- 1044

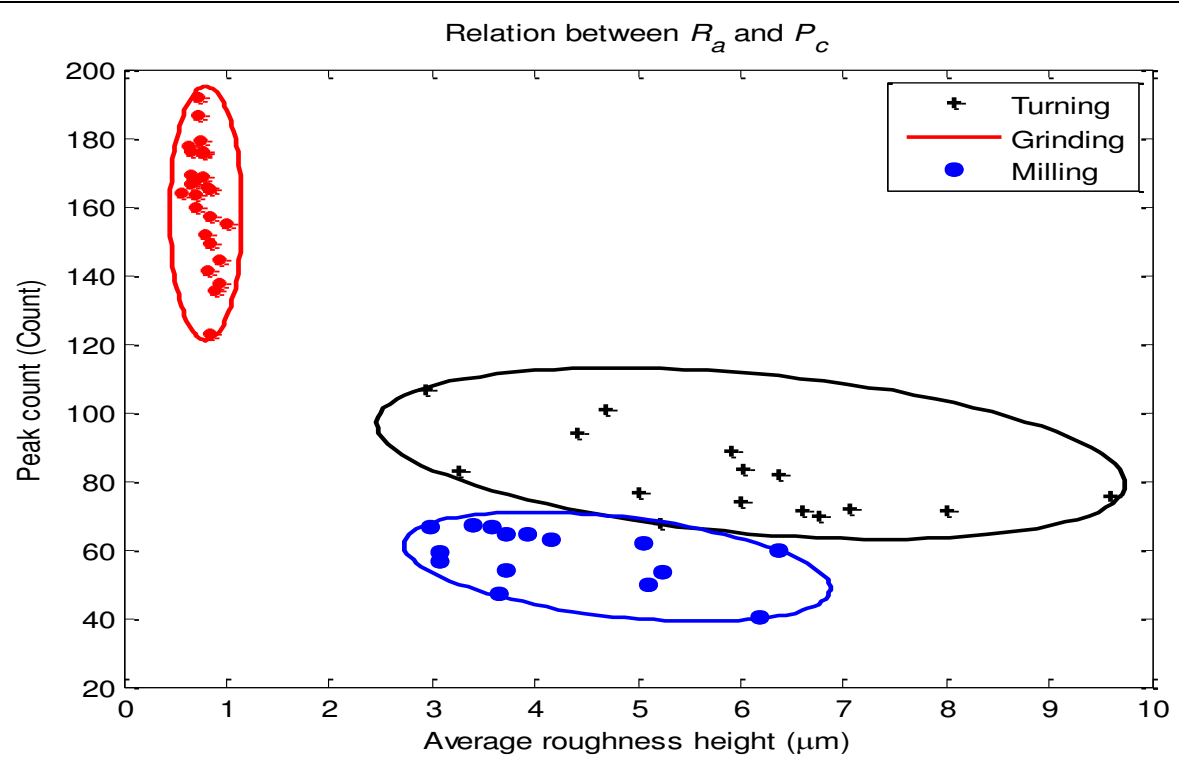

Fig. 6. A diagram of average roughness height $\left(R_{a}\right)$ versus peak count $\left(P_{c}\right)$ of typical machining operations

\section{Table 8.}

Ellipse parameters $\left(X_{c}, Y_{c}, a, b\right.$ and $\left.\varphi\right)$.

\begin{tabular}{ccccccc}
\hline \hline \multirow{2}{*}{ Parameter } & \multicolumn{2}{c}{ Turning (Facing) } & \multicolumn{2}{c}{ Milling } & \multicolumn{2}{c}{ Grinding } \\
$X_{c}$ & $R_{a}-S$ & $R_{a}-P_{c}$ & $R_{a}-S$ & $R_{a^{-}} P_{c}$ & $R_{a}-S$ & $R_{a^{-}} P_{c}$ \\
$Y_{c}$ & 0.8 & 0.8 & 4.8 & 6.1 & 6.1 & 4.8 \\
$a$ & 55 & 158 & 162 & 88 & 102 & 55 \\
$b$ & 0.28 & 0.35 & 1.8 & 3.4 & 3.3 & 1.9 \\
$\varphi$ & 15 & 37 & 46 & 25 & 30 & 16 \\
\hline \hline
\end{tabular}

\subsection{Analysis of cutting parameters}

The influences of machine parameters on machined parts are not always precisely known, and hence, it becomes difficult to recommend the optimum machinability data for machine process. Cutting parameter identification in machining operations, which included cutting speed, feed rate and depth of cut plays a very important role in the efficient utilization of machine tools and directly influences the product quality. Thus, it significantly influences the overall manufacturing costs. In practice, the machinists select cutting parameters from their specified ranges in machining handbooks, mainly based on experience, in order to satisfy the required accuracy of the final product [2]. The analysis of cutting parameters will be explained in details in section 4.2.

Journal of Engineering Sciences, Assiut University, Faculty of Engineering, Vol. 41, No. 3, May, 2013,E-mail address: jes@aun.edu.eg 
O. B. Abouelatta, Prediction of machining operations and surface roughness using artificial neural network, pp. 1021-1044

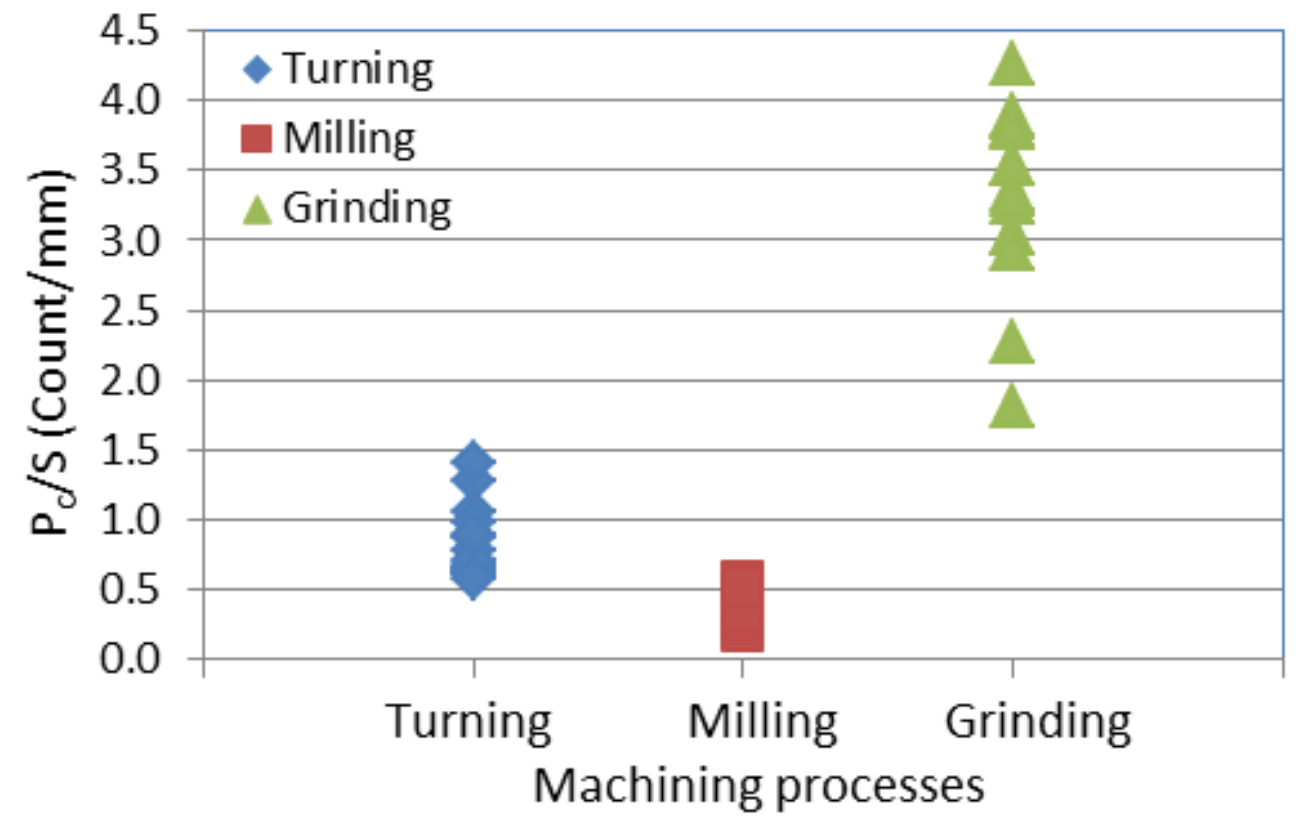

Fig. 7. Surface index $\left(P_{c} / S\right)$

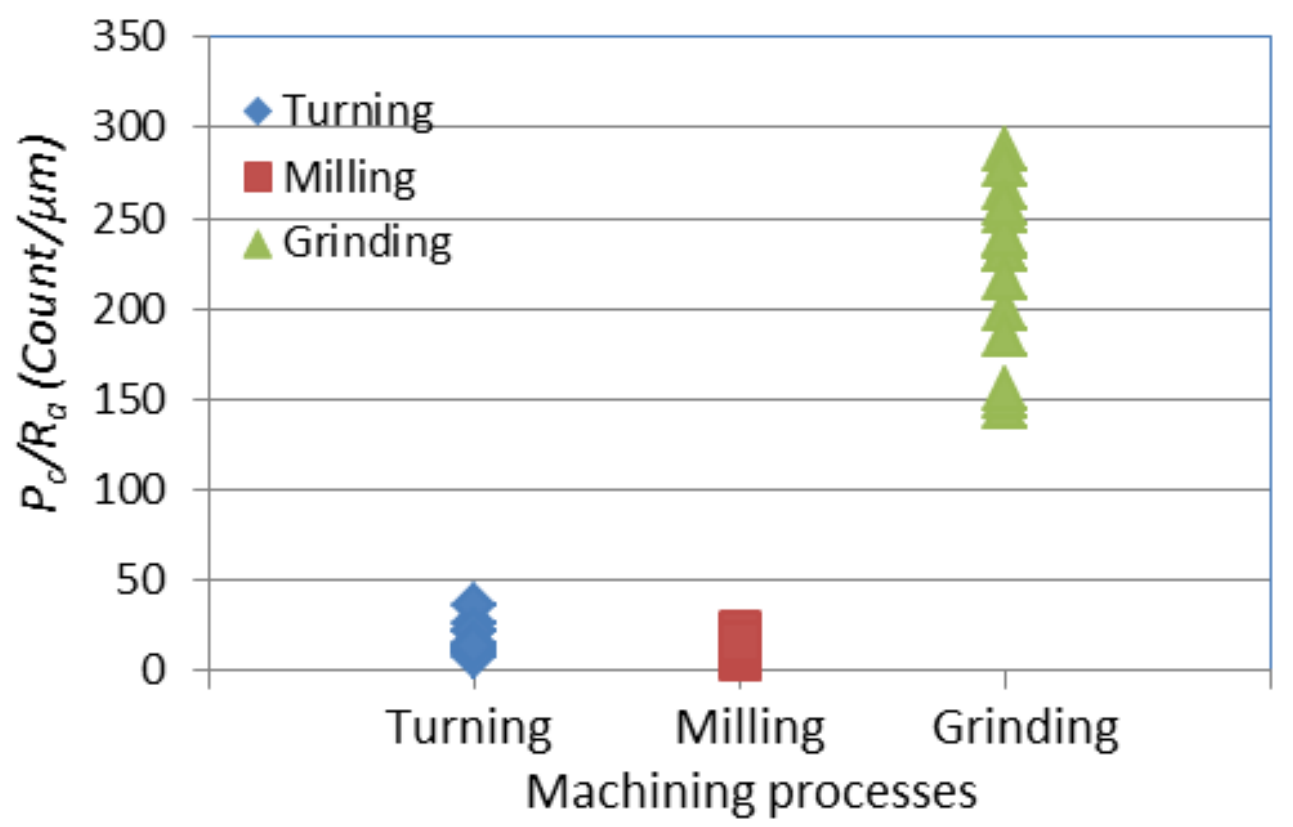

Fig. 8. Surface index $\left(P_{c} / R_{a}\right)$

Journal of Engineering Sciences, Assiut University, Faculty of Engineering, Vol. 41, No. 3, May, 2013,E-mail address: jes@aun.edu.eg 
O. B. Abouelatta, Prediction of machining operations and surface roughness using artificial neural network, pp. 1021- 1044

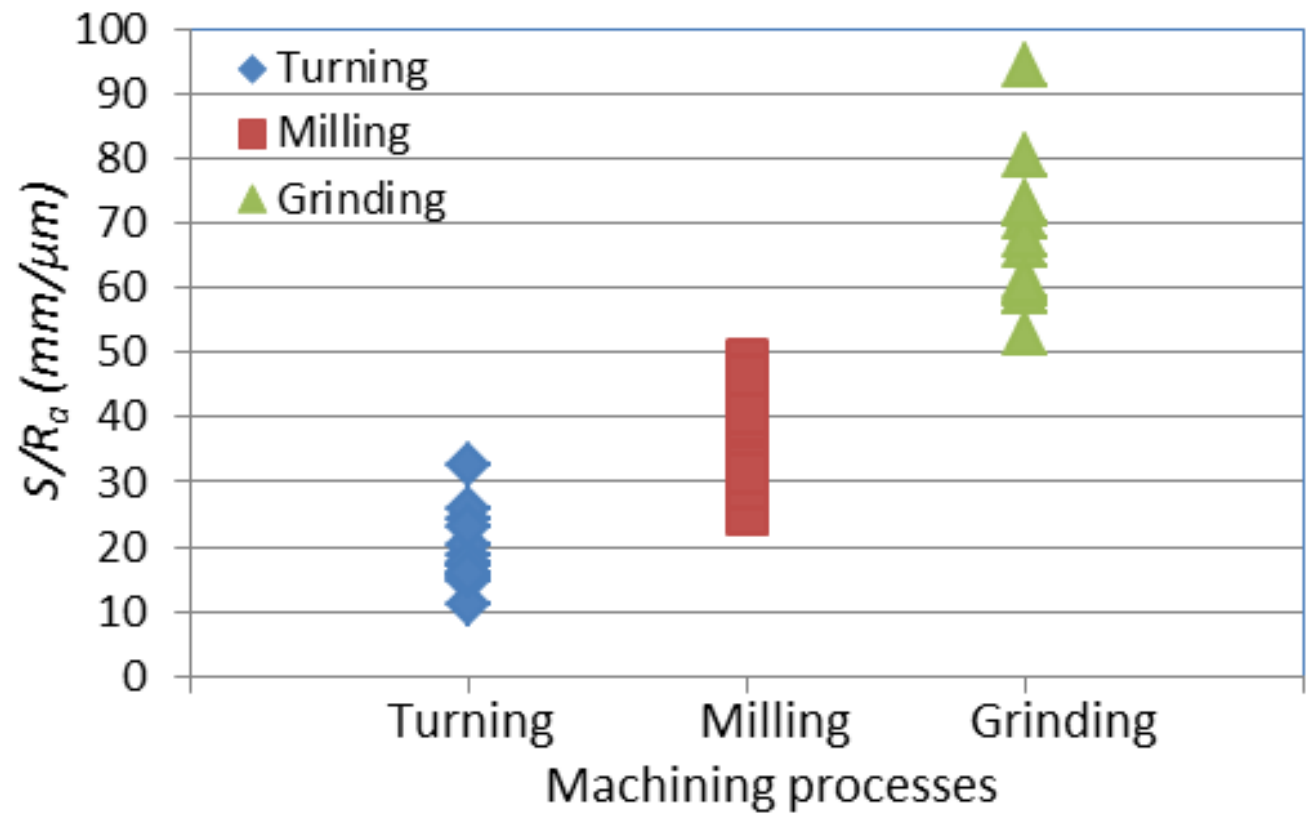

Fig. 9. Surface index $\left(S / R_{a}\right)$

\section{Neural network modeling}

Neural network is a highly flexible modeling tool with the ability to learn the mapping between input and output parameters. An artificial neural network (ANN) is capable of learning from an experimental data set to describe the nonlinear and interaction effects more effectively. The network consists of an input layer used to present data, output layer to produce ANN's response, and one or more hidden layers in between. The network is characterized by their topology, weight vectors, and activation function that are used in hidden and output layers of the network. Networks with biases, a sigmoid layer, and a linear output layer are capable of approximating any function with a finite number of discontinuities. The knowledge is presented by the interconnection weight, which is adjusted during the learning stage using the back propagation learning algorithm to minimize the mean square between the actual output of the network and the desired output pattern [23]. The overall training process used in this paper is illustrated in Fig. 10. Firstly, the roughness parameters were used as an input to predict the output which is the machining process. Secondly, both of roughness parameters and the predicted machining process were used to predict cutting parameters which are: feed, speed and depth of cut.

Journal of Engineering Sciences, Assiut University, Faculty of Engineering, Vol. 41, No. 3, May, 2013,E-mail address: jes@aun.edu.eg 


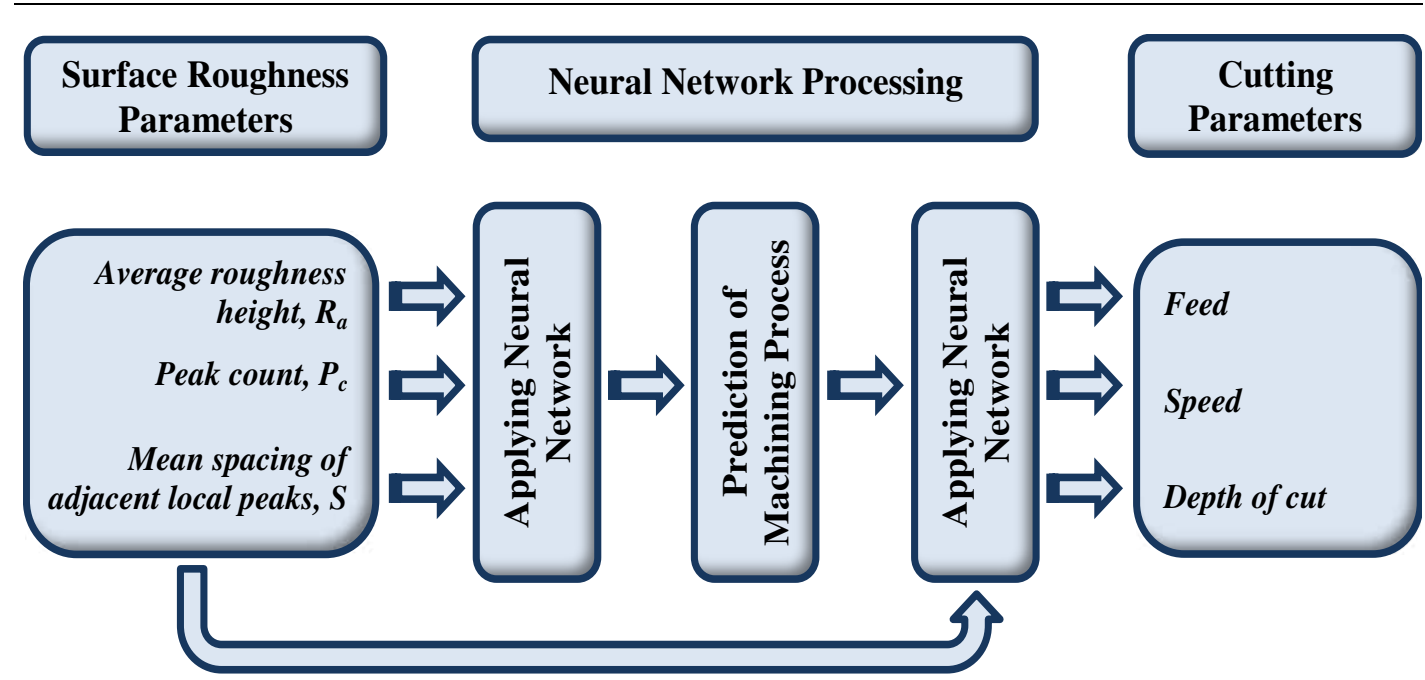

Fig. 10. Application of neural network to predict machining operations and cutting parameters

\subsection{Prediction of machining process}

A feed forward neural network with error back propagation algorithm was adopted for the NN system. Here it is used to develop machining process prediction model through surface roughness measurements. From 45 experiments were conducted, 36 experimental datasets were used to train the network. Before applying the neural network for modeling the architecture of the network has to be decided; i.e. the number of hidden layers and the number of neurons in each layer and transfer function for each layer. As there are three inputs $\left(R_{c}, P_{c}\right.$ and $S$ roughness parameters) to produce one output (Machining process which are: Turning "Facing", Milling or Grinding), the number of neurons in the input and output layer has to be set to 3 and 1 respectively as shown in Fig. 11. Considering one hidden layer, the number of neurons in the hidden layer is optimized. A procedure was employed to optimize the hidden layer neurons and select the transfer function for which a program was generated in MATLAB software. Accordingly, an experimental approach was adopted, which involves verification of the neural network against another 9 sets of data from 45 experimental dataset.

Journal of Engineering Sciences, Assiut University, Faculty of Engineering, Vol. 41, No. 3, May, 2013,E-mail address: jes@aun.edu.eg 


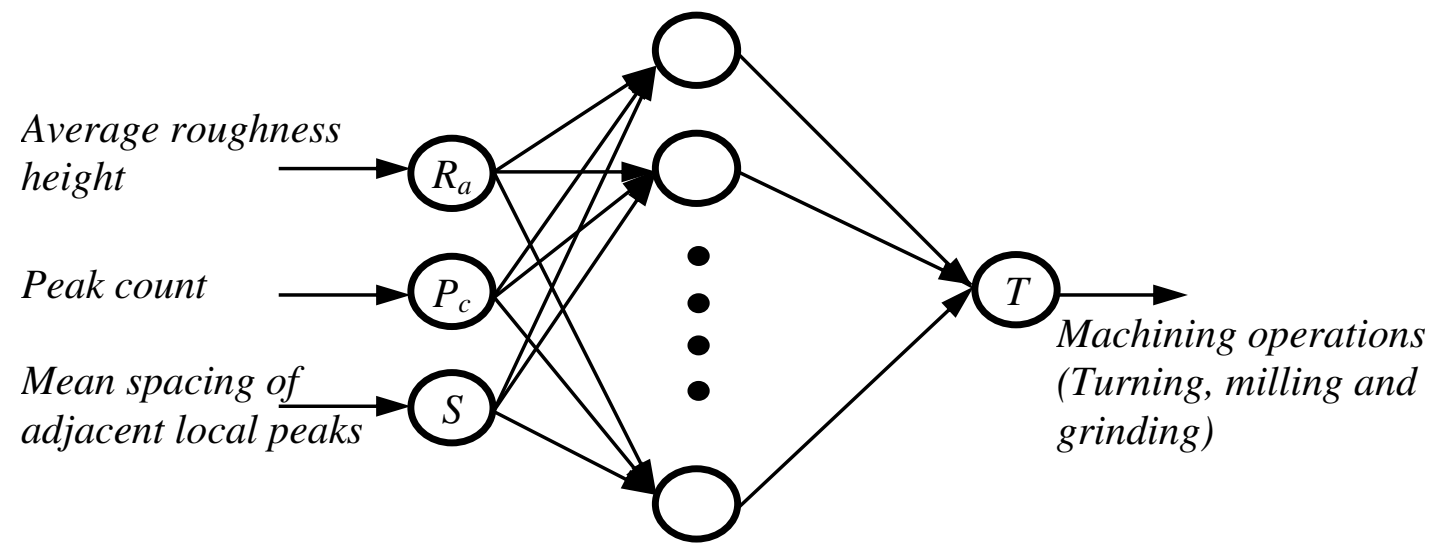

Input layer Hidden layer Output layer

Fig. 11. Application of neural network to predict machining operations

The performance of the network was evaluated by mean squared error between the experimental and the predicted values for every output nodes in respect of training the network. When the training is complete, the network performance could be checked and determined to search for if any changes need to be made to the training process, the network architecture or the data sets. The first thing to do is to check the training record, Fig. 12. As indicated, the iteration at which the validation performance reached a minimum was 6 . The training continued for 6 more iterations before the training stopped. The next step in validating the network is to create a regression plot, which shows the relationship between the outputs of the network and the targets, Fig. 13. If the training were perfect, the network outputs and the targets would be exactly equal, but the relationship is rarely perfect in practice. The four axes represent the training, validation, testing and all data. The dashed line in each axis represents the perfect result between outputs and targets. The solid line represents the best fit linear regression line between outputs and targets. The $R$ value is an indication of the relationship between the outputs and targets. If $R=1$, this indicates that there is an exact linear relationship between outputs and targets. If $R$ is close to zero, then there is no linear relationship between outputs and targets.

Journal of Engineering Sciences, Assiut University, Faculty of Engineering, Vol. 41, No. 3, May, 2013,E-mail address: jes@aun.edu.eg 


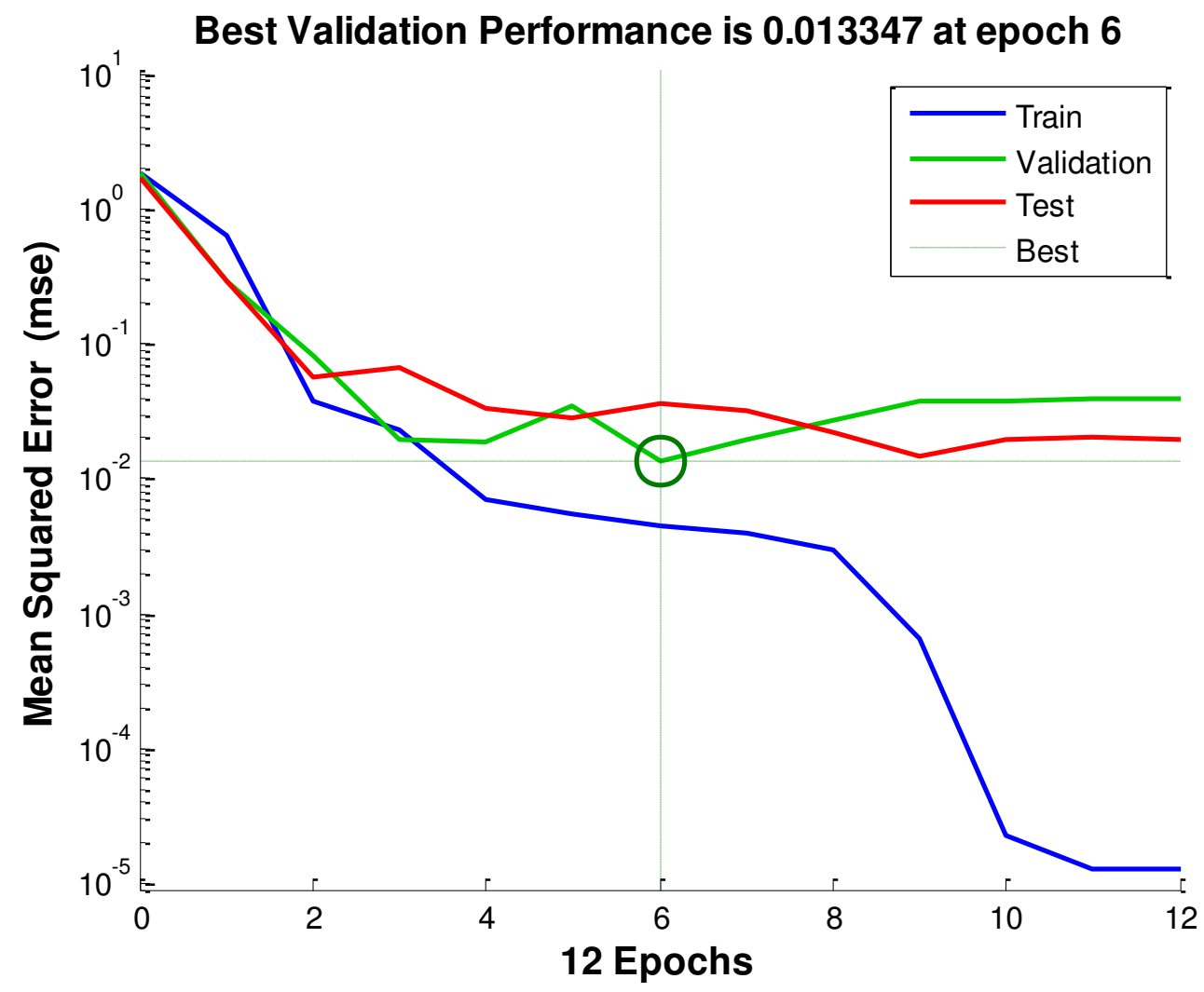

Fig.12. Plot of training, validation, and test performances

Table 9 shows the output of neural network process. The feedback from that processing is called the "average error" or "performance". Once the average error is below the required goal or reaches the required goal, the neural network stops training and becomes ready to be verified. This process was repeated one hundred times and the lowest MSE run was chosen as the best trained net. After the training process, the model is tested for validation. In this work, the network is validated in terms of agreement with an extra 9 experimental data listed in Tables 2-4.

Journal of Engineering Sciences, Assiut University, Faculty of Engineering, Vol. 41, No. 3, May, 2013,E-mail address: jes@aun.edu.eg 
O. B. Abouelatta, Prediction of machining operations and surface roughness using artificial neural network, pp. 1021- 1044
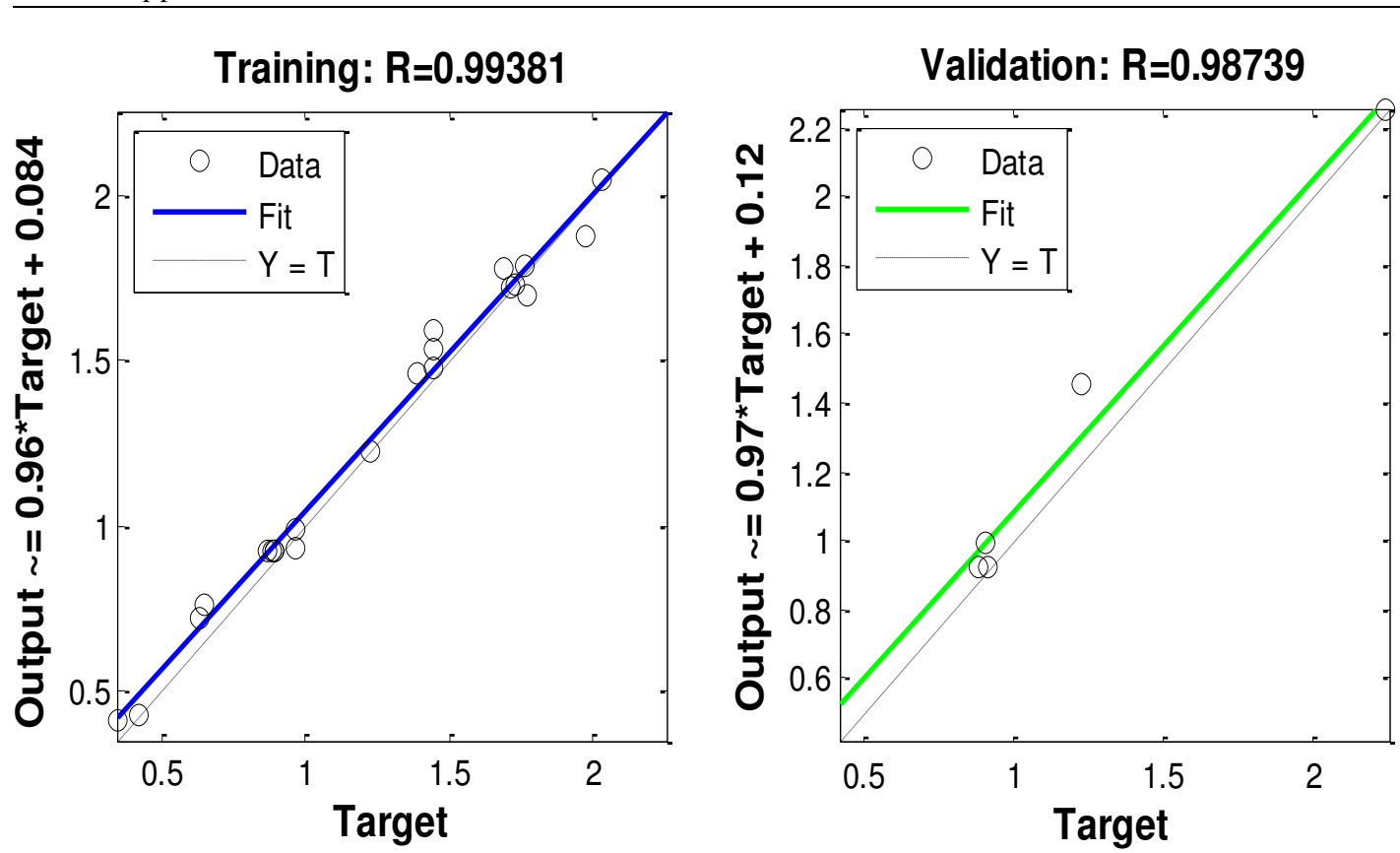

Test: $\mathbf{R}=\mathbf{0 . 8 9 0 9 4}$
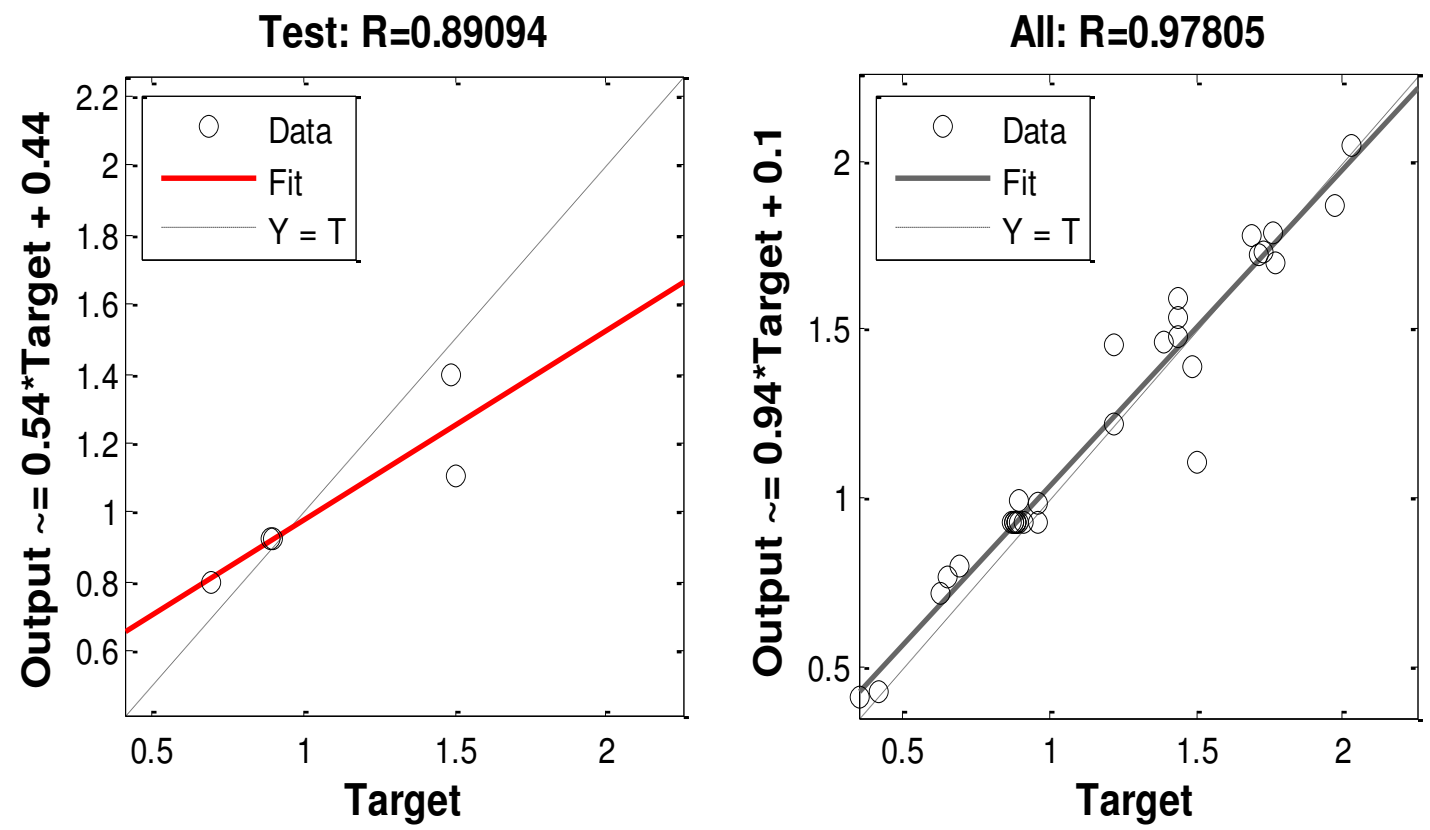

Fig. 13. Linear regression of targets relative to outputs

Journal of Engineering Sciences, Assiut University, Faculty of Engineering, Vol. 41, No. 3, May, 2013,E-mail address: jes@aun.edu.eg 
O. B. Abouelatta, Prediction of machining operations and surface roughness using artificial neural network, pp. 1021- 1044

\section{Table 9.}

Neural network output

\begin{tabular}{lccc}
\hline \hline \multicolumn{1}{c}{ Parameter } & Turning & Milling & Grinding \\
\hline Best trail number & 35 & 93 & 91 \\
Coefficient of determination $(R)$ & 0.9938 & 0.9092 & 0.93107 \\
Maximum error & 10.1929 & 92.3164 & 68.3333 \\
Average error & 2.43334 & 12.2651 & 5.2975 \\
Mean absolute error (MAE) & 0.02679 & 0.07882 & 0.04318 \\
Mean squared error (MSE) & 0.00187 & 0.02957 & 0.01111 \\
Mean squared normalized error (MSNE) & 1.91122 & 0.88089 & 4.1716 \\
Mean squared normalized error with & 1.92480 & 0.96344 & 4.0315 \\
regularization (MANEREG) & & & \\
Mean squared error with regularization & 0.20639 & 0.19726 & 0.28706 \\
(MAEREG) & & & \\
Mean squared error with regularization and & 0.10422 & 0.11489 & 0.14964 \\
economization (MSEREGEC) & 0.05983 & 0.79843 & 0.26664 \\
Sum squared error $($ SSE) & 1954 & 20464 & 22958 \\
Number of Iteration $(N I)$ & & & \\
\hline \hline
\end{tabular}

\subsection{Prediction of cutting parameters}

At first, an ANN model was proposed to determine surface roughness in a dry environment. The capability of the ANN model is to generalize unseen data depending on several factors. These factors are appropriate selection of input-output parameters, the distribution of the input-output dataset, and the format of the presentation of the dataset to the neural network. Selected input parameters are the significant variables that affect the surface roughness while perform machining operation under dry environment. Cutting speed, feed rate, and depth of cut were considered as input parameters. The output parameter of the model is the average roughness parameter $\left(R_{a}\right)$. Details of the input/output parameters of the proposed ANN model are illustrated in Fig. 14. [24]

Secondly, possible interaction cutting parameters (Feed, speed and depth of cut) were built and simulated using the trained net to calculate $R_{a}$. Searching for the nearest values to measured $R_{a}$ within 0.01 error, the possible cutting parameters that produce measured $R_{a}$ can be tabulated as shown in Prediction of Cutting Parameters Panel, Fig. 2.

The network architecture/ topology or features such as number of neurons and layers are very important factors that determine the functionality and generalization capability of the network. Training of an ANN plays a significant role in designing the direct ANN-based prediction. The same procedures were applied as in machining operation prediction. After the training, the model is tested for validation. In this work, the network is validated in terms of agreement with an extra 3 experimental data.

Journal of Engineering Sciences, Assiut University, Faculty of Engineering, Vol. 41, No. 3, May, 2013,E-mail address: jes@aun.edu.eg 
O. B. Abouelatta, Prediction of machining operations and surface roughness using artificial neural network, pp. 1021- 1044

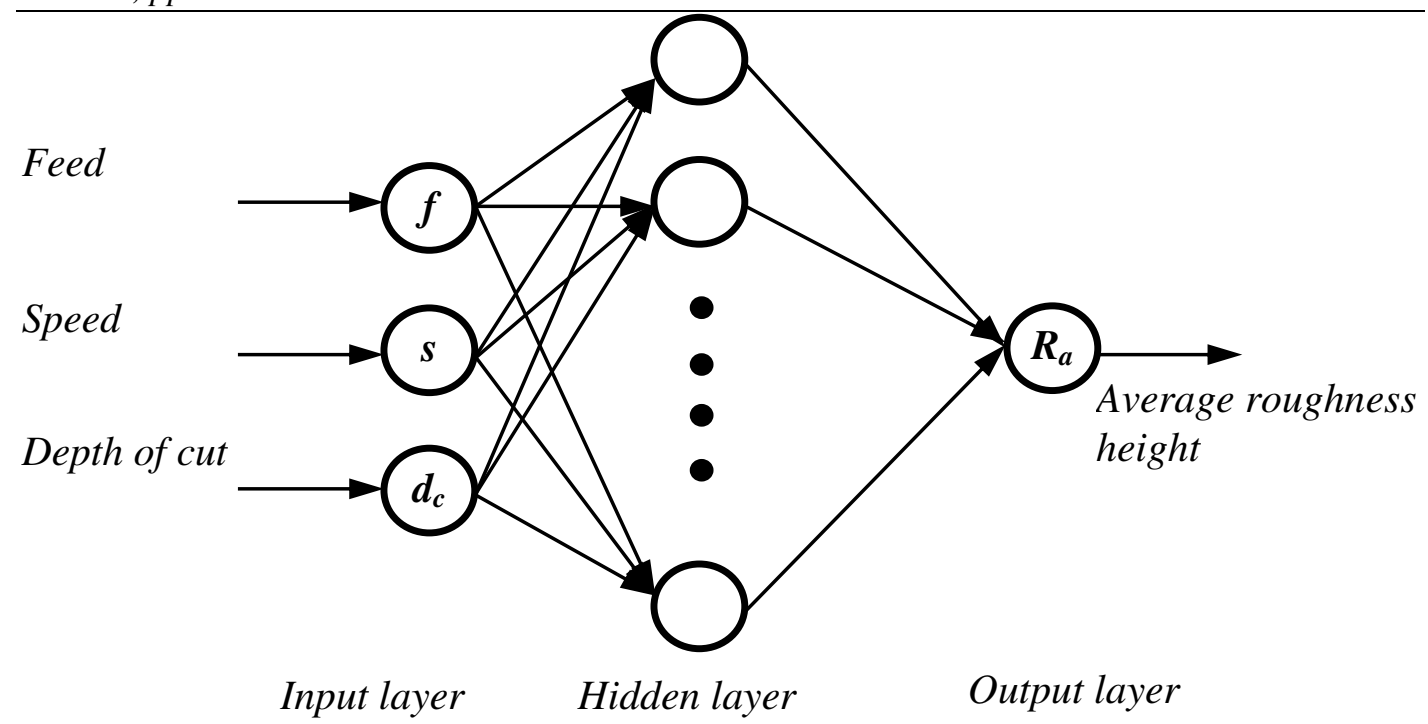

Fig. 14. Application of neural network to predict cutting parameters

\section{Conclusions}

In this study, an artificial neural network approaches were developed to predict the machining processes, surface roughness, and machining parameters while cutting AISI 1040 steel specimens. The obtained data were used to develop the surface roughness models. The capability of the developed prediction models were evaluated by comparing its results with measured values. The predicted values were found to be close to the measured values. The proposed models can be used effectively to predict the surface roughness in turning process. The coefficient of correlation $(R)$ is more than $90.9 \%$ for the testing data in neural network model. The following conclusions can be drawn from the present study.

1. Two-dimensional surface roughness parameters could be deduced to six groups. In other words, only six surface roughness parameters in $2 \mathrm{D}$ are enough to characterize machined surfaces.

2. The machining operations under investigations, which are turning (facing), milling and grinding; can be completely separated from each other using its surface roughness signature indexes.

3. Using inverse artificial neural network, it was possible to predict cutting parameters that produce a specified surface roughness values.

\section{References}

[1] Mohammed T. Hayajneh, Montasser S. Tahat, Joachim Bluhm, A Study of the Effects of Machining Parameters on the Surface Roughness in the End-Milling Process, Jordan Journal of Mechanical and Industrial Engineering, vol. 1, No. 1, pp. 1-5, 2007.

Journal of Engineering Sciences, Assiut University, Faculty of Engineering, Vol. 41, No. 3, May, 2013,E-mail address: jes@aun.edu.eg 
O. B. Abouelatta, Prediction of machining operations and surface roughness using artificial neural network, pp. 1021- 1044

[2] Adeel H. Suhail, N. Ismail, S. V. Wong and N. A. Abdul Jalil, "Cutting Parameters Identification using Multi Adaptive Network based Fuzzy Inference System: An Artificial Intelligence Approach", Scientific Research and Essays, Vol. 6, No. 1, pp. 187-195, 2011.

[3] ShutongXie, YinbiaoGuo, "Intelligent Selection of Machining Parameters in Multi-pass Turnings Using a GA-based Approach", Journal of Computational Information Systems, Vol. 7, No. 5, pp. 1714-1721, 2011.

[4] W. Grzesik, T. Wanat, "Comparative Assessment of Surface Roughness Produced by Hard Machining with Mixed Ceramic Tools Including 2D and 3D Analysis", Journal of Materials Processing Technology, Vol. 169, pp. 364-371, 2005.

[5] Rajendra M. Patrikar, "Modeling and Simulation of Surface Roughness", Applied Surface Science, Vol. 228, pp. 213-220, 2004.

[6] L.H.S. Luong, T.A. Spedding, "A Neural-Network System for Predicting Machining Behavior", Journal of Materials Processing Technology, Vol. 52, pp. 585-591, 1995

[7] A. Kohli and U.S. Dixit, "A Neural-Network-based Methodology for the Prediction of Surface Roughness in a Turning Process", Int J Adv Manuf Technol, Vol. 25, pp. 118-129, 2005.

[8] İlhan Asiltürk, Mehmet Çunkaş, "Modeling and Prediction of Surface Roughness in Turning Operations using Artificial Neural Network and Multiple Regression Method", Expert Systems with Applications, Vol. 38, pp. 5826-5832, 2011.

[9] İlhan Asiltürk, "Predicting Surface Roughness of Hardened AISI 1040 based on Cutting Parameters using Neural Networks and Multiple Regression", Int J Adv Manuf Technol, Published online: 05 February 2012.

[10] K. Sundara Murthy, I. Rajendran, "A Study on Optimisation of Cutting Parameters and Prediction of Surface Roughness in End Milling of Aluminium under MQL Machining", Int. J. Machining and Machinability of Materials, Vol. 7, Nos. 1/2, 2010.

[11]B. Huang and J. C. Chen, "An In-Process Neural Network-Based Surface Roughness Prediction (INN-SRP) System Using a Dynamometer in End Milling Operations", Int J Adv Manuf Technol, Vol. 21, pp. 339-347, 2003.

[12] Tuncay Erzurumlu, Hasan Oktem, "Comparison of Response Surface Model with Neural Network in Determining the Surface Quality of Moulded Parts", Materials and Design, Vol. 28, pp. 459-465, 2007.

[13] Antoni Wibowo, Mohammad Ishak Desa, "Kernel based Regression and Genetic Algorithms for Estimating Cutting Conditions of Surface Roughness in End Milling Machining Process", Expert Systems with Applications, Vol. 39, pp. 11634-11641, 2012.

[14] Nabil Ben Fredj, Ridha Amamou, "Ground Surface Roughness Prediction based upon Experimental Design and Neural Network Models", Int J Adv Manuf Technol, Vol. 31, pp. 24-36, 2006.

[15] Indrajit Mukherjee, Pradip Kumar Ray, "A Systematic Solution Methodology for Inferential Multivariate Modelling of Industrial Grinding Process", Journal of materials processing technology, Vol. 196, pp. 379-392, 2008.

[16] James J. Govindhasamy, Seán F. McLoone, George W. Irwin, John J. French, Richard P. Doyle, "Neural Modelling, Control and Optimisation of an Industrial Grinding Process", Control Engineering Practice, Vol. 13, pp. 1243-1258, 2005.

[17] Sanjeev Kumar, S.K. Choudhury, "Prediction of Wear and Surface Roughness in Electrodischarge Diamond Grinding", Journal of Materials Processing Technology, Vol. 191, pp. 206-209, 2007.

Journal of Engineering Sciences, Assiut University, Faculty of Engineering, Vol. 41, No. 3, May, 2013,E-mail address: jes@aun.edu.eg 
O. B. Abouelatta, Prediction of machining operations and surface roughness using artificial neural network, pp. 1021- 1044

[18]E. Mainsah and D.T. Ndumu, "Neural network applications in surface topography", Int. J. Mach. Tools Manufact. Vol. 38, Nos 5-6, pp. 591-598, 1998.

[19]E.S. Gadelmawla, M.M. Koura, T.M.A. Maksoud, I.M. Elewa, H.H. Soliman, "Surface Roughness", Journal of Materials Processing Technology, Vol. 123, pp. 133-145, 2002.

[20] D.J. Whitehouse, "The Parameter Rash — Is there a Cure?", Wear, Vol. 83, No. 1, pp. 7578, 1982.

[21] B.L. Griffiths, "Manufacturing Surface Design and Monitoring for Performance", Surface Topography, Vol. 1, No. 1, pp. 61-69, 1988.

[22] Besant, W.H., "Chapter III. The Ellipse", Conic Sections, London: George Bell and Sons, p. 50, 1907.

[23] Jignesh G. Parmar, Alpesh Makwana, "Prediction of Surface Roughness for End Milling Process using Artificial Neural Network", International Journal of Advanced Engineering Research and Studies, E-ISSN2249-8974, IJAERS, Vol. I, Issue III, pp. 47-50,April-June, 2012.

[24] Diwakar Reddy V., Krishnaiah G., A. Hemanth Kumar and Sushil K. Priya, "ANN based Prediction of Surface Roughness in Turning", International Conference on Trends in Mechanical and Industrial Engineering (ICTMIE'2011) Bangkok, pp. 165-170, 2011.

\section{التنبؤ بعمليات التشغيل وخشونة الأسطح باستخدام الشبكة العصبية الاصطناعية}

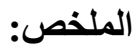

أصبحت خشونة السطح باعتبار ها واحدة من المتطلبات المهمة للعملاء و التي على أساسها يتم

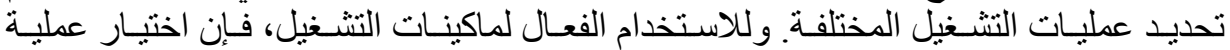

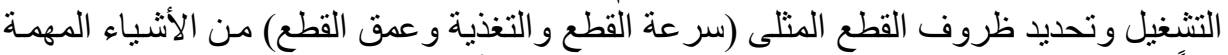

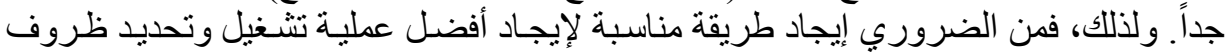

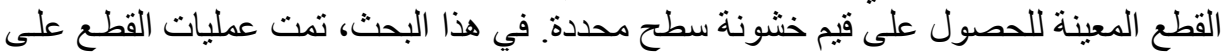

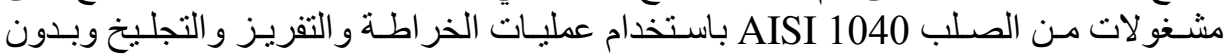

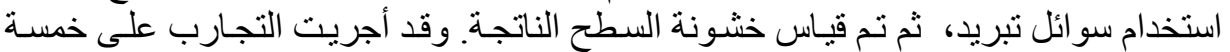

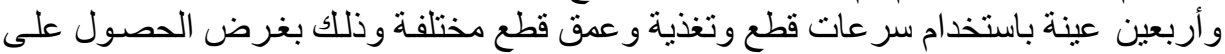

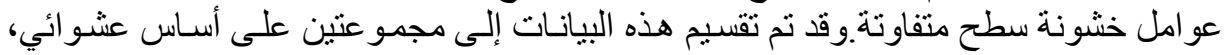
منها 36 عينة لمجمو عة بيانات التدريب و 9 لمجمو عة بيانات الاختبار . وقد استخدمت مجمو عة المة البيانـات التدريب لتدريب نمـاذج من الشبكات العصبية الاصطية التصاعية المختلفـة (ANN) للتنبؤ

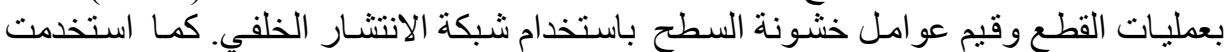

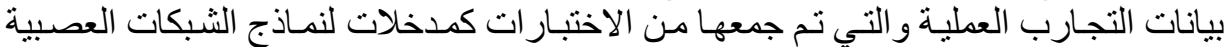

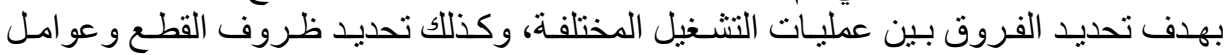

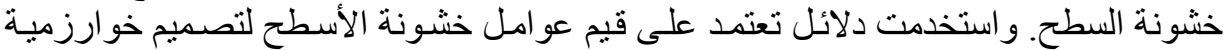

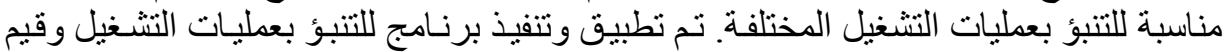

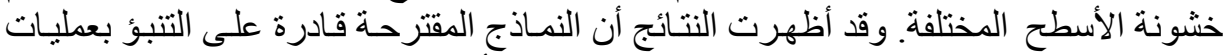
النتشغيل وتحديد ظروف القطع وكذلك تعيين قيم خشونة الأسطح المختلفة.

Journal of Engineering Sciences, Assiut University, Faculty of Engineering, Vol. 41, No. 3, May, 2013,E-mail address: jes@aun.edu.eg 